\title{
TORI IN THE CREMONA GROUPS
}

\author{
VLADIMIR L. POPOV*
}

\begin{abstract}
We classify up to conjugacy the subgroups of certain types in the full, in the affine, and in the special affine Cremona groups. We prove that the normalizers of these subgroups are algebraic. As an application, we obtain new results in the Linearization Problem generalizing to disconnected groups Białynicki-Birula's results of $1966-67$. We prove "fusion theorems" for $n$-dimensional tori in the affine and in the special affine Cremona groups of rank $n$. In the final section we introduce and discuss the notions of Jordan decomposition and torsion primes for the Cremona groups.
\end{abstract}

\section{Contents}

1. Introduction

2. Some subgroups of $\mathrm{Cr}_{n}$

3. Tori in $\mathrm{Cr}_{n}$, Aut $\mathbf{A}^{n}$, and $\mathrm{Aut}^{*} \mathbf{A}^{n} \quad 10$

4. Orbits and stabilizers of the action of $D_{n}\left(l_{1}, \ldots, l_{n}\right)$ on $\mathbf{A}^{n} \quad 13$

5. The group $N_{\text {Aut }} \mathbf{A}^{n}\left(D_{n}\left(l_{1}, \ldots, l_{n}\right)\right) \quad 16$

6. Fusion theorems for tori in Aut $\mathbf{A}^{n}$ and Aut ${ }^{*} \mathbf{A}^{n} \quad 22$

7. Applications: The classifications of classes of conjugate subgroups 23

8. Jordan decomposition in $\mathrm{Cr}_{n}$. Torsion primes for the Cremona groups 26

$\begin{array}{ll}\text { References } & 29\end{array}$

\section{INTRODUCTION}

This work arose from the attempt to solve the problem posed in [Po05 $]$, $\left[\mathrm{Po}_{2} 5_{2}\right]$. In these papers have been introduced the notions of root $\alpha$ and root vector $D$ of an affine algebraic variety $X$ with respect to an algebraic torus $T \subseteq$ Aut $X$. Namely, $D$ is a locally-nilpotent derivation of the coordinate algebra of the variety $X$, and $\alpha$ is a character of the torus $T$ such that $t^{*} \circ$ $D \circ t^{*-1}=\alpha(t) D$ for all $t \in T$. These definitions are inspired by the natural analogy with the classical definitions of the theory of algebraic groups and

\footnotetext{
* Supported by grants РФФИ 11-01-00185-a, НШ-5139.2012.1, and the program Contemporary Problems of Theoretical Mathematics of the Russian Academy of Sciences, Branch of Mathematics.
} 
purport an attempt to apply to, in general, infinite dimensional group Aut $X$ the techniques important in the theory of usual algebraic groups ${ }^{1}$.

In $\left[\mathrm{Po} 05_{1}\right],\left[\mathrm{Po}_{2}\right]$ the following two problems related to the classical case where $X=\mathbf{A}^{n}$ and $T=D_{n}^{*}$ is the maximal diagonal torus that leaves fixed the standard volume form (see below (9)) have been posed:

(R) Find all the roots and root vectors of the variety $\mathbf{A}^{n}$ with respect to $D_{n}^{*}$.

(W) Describe the normalizer and centralizer of the torus $D_{n}^{*}$ in the group Aut $^{*} \mathbf{A}^{n}$ of automorphisms of the space $\mathbf{A}^{n}$ that leave fixed the standard volume form.

Problem (R) has been solved by Liendo in [Li11]. The answer is the following. Let $x_{1}, \ldots, x_{n}$ be the standard coordinate functions on $\mathbf{A}^{n}$ and let $\varepsilon_{1}, \ldots, \varepsilon_{n}$ be the "coordinate" characters of the standard $n$-dimensional diagonal torus $D_{n}$ in Aut $\mathbf{A}^{n}$ (see below (8) and (10)). Then, up to multiplication by a nonzero constant, the root vectors are precisely all the derivations $D$ of the form

$$
x_{1}^{l_{1}} \cdots x_{n}^{l_{n}}\left(\partial / \partial x_{i}\right),
$$

where $l_{1}, \ldots, l_{n}$ are nonnegative integers and $l_{i}=0$. The root $\alpha$ corresponding to root vector (1) is the restriction to $D_{n}^{*}$ of the character

$$
\varepsilon_{i}^{-1} \prod_{j=1}^{n} \varepsilon_{j}^{l_{j}} .
$$

The problem mentioned in the beginning of this introduction is Problem (W). It is clear that it is aimed at getting a description of the "Weyl group" of the root system from Problem (R). We solve it in the present paper. Namely, we prove (Theorem 14) that the normalizer (centralizer) of the torus $D_{n}^{*}$ in Aut $^{*} \mathbf{A}^{n}$ coincides with its normalizer (centralizer) in $\mathrm{SL}_{n}$, so that the Weyl group of $D_{n}^{*}$ in $\mathrm{Aut}^{*} \mathbf{A}^{n}$ is the same as that of $D_{n}^{*}$ in $\mathrm{SL}_{n}$ - it is the group of all permutations of the characters $\varepsilon_{1}, \ldots, \varepsilon_{n}$.

In fact, this result is only one special case of the series of general results that we obtain here. Namely, $D_{n}^{*}$ is only one of the infinitely many nonconjugate diagonalizable algebraic subgroups $G$ of dimension $\geqslant n-1$ in the group Aut $\mathbf{A}^{n}$. We prove that the normalizer of $G$ in Aut $\mathbf{A}^{n}$ always is an algebraic subgroup in Aut $\mathbf{A}^{n}$ (Theorem 16). It is the characteristic property of the specified dimensions: in general, for the diagonalizable subgroups of dimension $\leqslant n-2$ it does not hold. Moreover, in the case when nonconstant $G$-invariant polynomial functions on $\mathbf{A}^{n}$ exist, we explicitly describe the normalizer of $G$ in Aut $\mathbf{A}^{n}$, in particular, we show that in all but one cases it coincides with the normalizer of $G$ in a group conjugate to $\mathrm{GL}_{n}$ (Theorem 6).

Using the found information, we obtain the new results in the Linearization Problem. In 1966-67 Białynicki-Birula proved [B-B66], [B-B67] that every algebraic action on $\mathbf{A}^{n}$ of an algebraic torus of dimension $\geqslant n-1$ is equivalent to a linear action. We extend this statement to disconnected groups proving that every algebraic action on $\mathbf{A}^{n}$ of either an $n$-dimensional

\footnotetext{
${ }^{1} \operatorname{In}\left[\mathrm{Po} 05_{1}\right],\left[\mathrm{Po}_{2}\right]$ was considered the case where $X=\mathbf{A}^{n}$ and $T$ is the maximal diagonal torus that leaves fixed the standard volume form, but this restriction plays no role in the definition.
} 
algebraic group whose connected component of identity is a torus, or an $(n-1)$-dimensional diagonalizable group is equivalent to a linear action (Theorems 13 and 15).

We also obtain the following classifications:

(i) the classification of diagonalizable subgroups of the group $\operatorname{Aff}_{n}$ of affine transformastions (see below (6)) up to conjugacy in the full Cremona group $\mathrm{Cr}_{n}=\operatorname{Bir} \mathbf{A}^{n}$ (Theorem 1);

(ii) the classification of $n$-dimensional diagonalizable subgroups of Aut $\mathbf{A}^{n}$ up to conjugacy in Aut $\mathbf{A}^{n}$ (Theorem 11);

(iii) the classification of $(n-1)$-dimensional diagonalizable subgroups of Aut $^{*} \mathbf{A}^{n}$ up to conjugacy in Aut* $\mathbf{A}^{n}$ (Theorem 12);

(iv) the classiciation, up to conjugacy in Aut $\mathbf{A}^{n}$, of maximal $n$-dimensional algebraic subgroups $G$ in Aut $\mathbf{A}^{n}$ such that $G^{0}$ is a torus (Theorem 11);

(v) the classiciation, up to conjugacy in Aut $^{*} \mathbf{A}^{n}$, of maximal $(n-1)$ dimensional algebraic subgroups $G$ in Aut* $\mathbf{A}^{n}$ such that $G^{0}$ is a torus (Theorem 14);

(vi) the classification of $(n-1)$-dimensional diagonalizable subgroups of Aut $\mathbf{A}^{n}$ up to conjugacy in Aut $\mathbf{A}^{n}$ (Theorem 15);

(vii) the classification of diagonalizable subgroups in Aut $\mathbf{A}^{n}$ of dimension $\geqslant n-1$ up to conjugacy in $\mathrm{Cr}_{n}$ (Theorems 11 and 17);

(viii) the classification of one-dimensional tori of Aut $\mathbf{A}^{3}$ up to conjugacy in Aut $\mathbf{A}^{3}$ (Theorem 18).

For instance, we prove that the set of classes of $(n-1)$-dimensional diagonalizable subgroups of Aut $\mathbf{A}^{n}$ that are conjugate in Aut $\mathbf{A}^{n}$ is bijectively parametrized by the set of nonzero nondecreasing sequences

$$
\left(l_{1}, \ldots, l_{n}\right) \in \mathbf{Z}^{n},
$$

such that $\left(l_{1} \ldots, l_{n}\right) \leqslant\left(-l_{n} \ldots,-l_{1}\right)$ with respect to the lexicographic order. Under this parametrization, to sequence (2) corresponds the class of the subgroup ker $\varepsilon_{1}^{l_{1}} \cdots \varepsilon_{n}^{l_{n}}$.

Another example: we show that diagonalizable subgroups of Aff $_{n}$ are conjugate in $\mathrm{Cr}_{n}$ if and only if they are isomorphic and we specify their canonical representatives. In particular (see Corollary 5), every two isomorphic finite Abelian subgroups of $\mathrm{Aff}_{n}$ are conjugate in $\mathrm{Cr}_{n}$ (for finite cyclic subgroups this has been proved in $\left.\left[\mathrm{Bl} 106_{1}\right]\right)$.

In [Se10] Serre proved "fusion theorem" for the torus $D_{n}$ in $\mathrm{Cr}_{n}$. We prove and use "fusion theorems" for $n$-dimensional tori in Aut $\mathbf{A}^{n}$ and in Aut $^{*} \mathbf{A}^{n+1}$ (Theorem 9).

In the final section, developing further the theme of analogies between the Cremona groups and algebraic groups, we introduce and discuss the notions of Jordan decomposition and torsion primes for the Cremona groups. In the course of discussion, we formulate some open questions.

Acknowledgment. I am grateful to J.-P. Serre for the comments. 


\section{Notation and conventions.}

In the sequel, "variety" means "algebraic variety over the fixed algebraically closed field $k$ of characteristic zero" in the sense of Serre's FAC [Se55]. Apart from the standard notation and conventions of [Bo91] and [PoVi94] used without reminders we also use the following:

- $\operatorname{Mat}_{m \times n}(R)$ is the set of all matrices with $m$ rows, $n$ columns, and the coefficients in $R$.

- $N_{H}(S)$ and $Z_{H}(S)$ are, respectively, the normalizer and centralizer of the subgroup $S$ of the group $H$.

- $\boldsymbol{\mu}_{d}$ is the subgroups of order $d$ in $\mathbf{G}_{\mathrm{m}}$.

- $\mathrm{X}(D)$ is the group of rational characters of the diagonalizable algebraic group $D$.

- $\chi(X)$ is the Euler characteristic of the variety $X$ relative to the $l$ adic cohomology (for $k=\mathbf{C}$, by [La81] it coincides with the Euler characteristic relative to the usual cohomology with compact supports (cf. also [KrPo85, Appendix])).

- If the group $G$ acts on a set $M$ and $\varphi: G \times M \rightarrow M$ is the map defining this action, then for the subsets $S \subseteq G$ and $X \subseteq M$, the subset $\varphi(S \times X) \subseteq M$ is denoted by $S \cdot X$ (each time it is clear from the context what $\varphi$ is meant). In particular, by $G \cdot a$ is denoted the $G$-orbit of the point $a$. By $G_{a}$ is denoted the $G$-stabilizer of the point $a$.

- $x_{1}, \ldots, x_{n}$ are the standard coordinate functions on $\mathbf{A}^{n}$ :

$$
x_{i}(a):=a_{i}, \quad a:=\left(a_{1}, \ldots, a_{n}\right) \in \mathbf{A}^{n} .
$$

In the sequel it is assumed that all considered algebraic groups are affine and all their homomorphisms are algebraic. Below all tori and diagonalizable groups are algebraic.

An action of a group $G$ on a vector space $V$ is called locally finite, if for every vector $v \in V$ the linear span of the orbit $G \cdot v$ is finite dimensional.

The group $\mathrm{Cr}_{n}:=\operatorname{Bir} \mathbf{A}^{n}$ is called the Cremona group of rank $n$. The map $\varphi \mapsto\left(\varphi^{*}\right)^{-1}$ identifies it with $\operatorname{Aut}_{k} k\left(x_{1}, \ldots, x_{n}\right)$. Every birational isomorphism $X \rightarrow \mathbf{A}^{n}$ identifies $\mathrm{Cr}_{n}$ with Bir $X$. For every $g \in \mathrm{Cr}_{n}$ the functions

$$
g_{i}=g^{*}\left(x_{i}\right) \in k\left(\mathbf{A}^{n}\right)
$$

determine $g$ by the formula

$$
g(a)=\left(g_{1}(a), \ldots, g_{n}(a)\right) \text { if } g \text { is defined at } a \in \mathbf{A}^{n} ;
$$

we use the notation

$$
\left(g_{1}, \ldots, g_{n}\right):=g
$$

By means of the notion of "algebraic family" $S \rightarrow \mathrm{Cr}_{n}$ (see [Ra64]) the group $\mathrm{Cr}_{n}$ is endowed with the Zariski topology (see [Se10], [Bl10]). If a homomorphism $G \rightarrow \mathrm{Cr}_{n}$ of an algebraic group $G$ is an algebraic family, then its image is called an algebraic subgroup of $\mathrm{Cr}_{n}$ (see [Po12]).

The subgroup

$$
\text { Aut } \mathbf{A}^{n}:=\left\{\left(g_{1}, \ldots, g_{n}\right) \in \mathrm{Cr}_{n} \mid g_{1}, \ldots, g_{n} \in k\left[\mathbf{A}^{n}\right]=k\left[x_{1}, \ldots, x_{n}\right]\right\}
$$


is called the affine Cremona group of rank $n$.

It contains the algebraic subgroup of affine transformations

$$
\operatorname{Aff}_{n}=\left\{\left(g_{1}, \ldots, g_{n}\right) \in \operatorname{Aut} \mathbf{A}^{n} \mid \operatorname{deg} g_{1}=\ldots=\operatorname{deg} g_{n}=1\right\},
$$

and $\mathrm{Aff}_{n}$, in turn, contains the algebraic subgroup of linear transformations

$$
\mathrm{GL}_{n}=\left\{g \in \mathrm{Aff}_{n} \mid g(0)=0\right\} .
$$

If $g=\left(g_{1}, \ldots, g_{n}\right) \in \operatorname{Aut} \mathbf{A}^{n}$ (see (5)), then we put

$$
\operatorname{Jac}(g):=\operatorname{det}\left(\partial g_{i} / \partial x_{j}\right) \text {. }
$$

Since $g \in \operatorname{Aut} \mathbf{A}^{n}$, we have $\operatorname{Jac}(g) \in k \backslash\{0\}$. Therefore, $g \mapsto \operatorname{Jac}(g)$ is the homomorphism of Aut $\mathbf{A}^{n}$ in the multiplicative group of the field $k$. Its kernel

$$
\text { Aut }^{*} \mathbf{A}^{n}:=\left\{f \in \operatorname{Aut} \mathbf{A}^{n} \mid \operatorname{Jac}(g)=1\right\}
$$

consists of the automorphisms of $\mathbf{A}^{n}$ that leaves fixed the standard volume form; it is called the special affine Cremona group of rank $n-1$ (regarding the ranks in these names see Theorems 2(i) and 4(i) below). The latter group contains the algebraic subgroup

$$
\mathrm{SL}_{n}:=\mathrm{GL}_{n} \cap \mathrm{Aut}^{*} \mathbf{A}^{n} \text {. }
$$

The embeddings $\mathrm{Cr}_{n} \hookrightarrow \mathrm{Cr}_{n+1},\left(g_{1}, \ldots, g_{n}\right) \mapsto\left(g_{1}, \ldots, g_{n}, x_{n+1}\right)$ are arranged the tower $\mathrm{Cr}_{1} \hookrightarrow \mathrm{Cr}_{2} \hookrightarrow \cdots \hookrightarrow \mathrm{Cr}_{n} \hookrightarrow \cdots$. Its direct limit $\mathrm{Cr}_{\infty}$ is called the Cremona group of infinite rank, see [Po12, Sect.1].

In $\mathrm{GL}_{n}$ is distinguished the "standard" maximal torus

$$
D_{n}:=\left\{\left(t_{1} x_{1}, \ldots t_{n} x_{n}\right) \mid t_{1}, \ldots, t_{n} \in k\right\} \subset \mathrm{GL}_{n} .
$$

Its normilizer in $\mathrm{GL}_{n}$ is the group of all monomial transformations in $\mathrm{GL}_{n}$ :

$$
N_{\mathrm{GL}_{n}}\left(D_{n}\right)=\left\{\left(t_{1} x_{\sigma(1)}, \ldots, t_{n} x_{\sigma(n)}\right) \mid \sigma \in S_{n}, t_{1}, \ldots, t_{n} \in k\right\} \subset \mathrm{GL}_{n},
$$

where $S_{n}$ is the symmetric group of degree $n$. In Aut $^{*} \mathbf{A}^{n}$ is contained the torus

$$
D_{n}^{*}:=D_{n} \cap \mathrm{Aut}^{*} \mathbf{A}^{n}=\left\{\left(t_{1} x_{1}, \ldots, t_{n} x_{n}\right) \mid t_{1}, \ldots, t_{n} \in k, t_{1} \cdots t_{n}=1\right\} .
$$

The "coordinate" characters $\varepsilon_{1}, \ldots, \varepsilon_{n}$ of the torus $D_{n}$ defined by

$$
\varepsilon_{i}: D_{n} \rightarrow \mathbf{G}_{\mathrm{m}}, \quad\left(t_{1} x_{1}, \ldots, t_{n} x_{n}\right) \mapsto t_{i},
$$

constitute a base of the (free Abelian) group $\mathrm{X}\left(D_{n}\right)$.

\section{Some SubGroups of $\mathrm{Cr}_{n}$}

In the sequel we consider the elements of the group $\mathbf{Z}^{n}$ as rows of length $n$. Then the rows of any matrix $A=\left(a_{i j}\right) \in \operatorname{Mat}_{m \times n}(\mathbf{Z})$ become the elements of this group and we use the following notation:

$\mathcal{R}_{A}:=$ the subgroup of $\mathbf{Z}^{n}$ generated by the rows of matrix $A$,

$$
D_{n}(A)=\bigcap_{i=1}^{m} \operatorname{ker} \lambda_{i}, \quad \text { where } \quad \lambda_{i}:=\varepsilon_{1}^{a_{i, 1}} \cdots \varepsilon_{n}^{a_{i, n}} .
$$


If $m=1$, then in place of $D_{n}\left(\left(l_{1} \ldots l_{n}\right)\right)$ we write $D_{n}\left(l_{1}, \ldots, l_{n}\right)$. In particular,

$$
D_{n}(0, \ldots, 0)=D_{n} .
$$

Clearly, $D_{n}(A)$ is a closed subgroup of $D_{n}$ and (see (11))

$$
D_{n}(A)=\bigcap_{\left(l_{1}, \ldots, l_{n}\right) \in \mathcal{R}(A)} \operatorname{ker} \varepsilon_{1}^{l_{1}} \cdots \varepsilon_{n}^{l_{n}} .
$$

Recall the terminology used below (see, e.g., [Vi03] and [MaMi64]).

Every finite Abelian group $G$ decomposes as a direct sum of cyclic subgroups of orders $d_{1}, \ldots, d_{m}$, where $d_{i}$ divides $d_{i+1}$ for $i=1, \ldots, m-1$, and $d_{1}>1$ if $|G|>1$. The numbers $d_{1}, \ldots, d_{s}$ are uniquely determined by $G$ and are called the invariant factors of $G$.

Every nonzero integer matrix $A$ can be transformed by means of elementary transformations of its rows and columns into a matrix $S=\left(s_{i j}\right)$ such that only the coefficients $s_{i i}$ for $i=1, \ldots, r$ are nonzero and $s_{i i}$ divides $s_{i+1, i+1}$ for $i=1, \ldots, r-1$. The integers $s_{11}, \ldots, s_{r r}$ are uniquely determined by $A$ $\left(_{i i}=f_{i} / f_{i-1}\right.$, where $f_{i}$ is gcd of the minors of order $i$ of the matrix $A$ and $\left.f_{0}:=1\right)$ and are called the invariant factors of matrix $A$. The matrix $S$ is called the Smith normal form of matrix A.

Lemma 1. If $B$ is obtained from $A \in \operatorname{Mat}_{m \times n}(\mathbf{Z})$ by means of the elementary transformations of rows and columns, then the subgroups $D_{n}(A)$ and $D_{n}(B)$ are conjugate in $\mathrm{Cr}_{n}$.

Proof. Let $\tau_{1}, \ldots, \tau_{n}$ be some base of the group $\mathrm{X}\left(D_{n}\right)$. Then (see (12)) $\lambda_{i}=\tau_{1}^{c_{i, 1}} \cdots \tau_{n}^{c_{i, n}}$ for some $c_{i j} \in \mathbf{Z}$ and

$$
D_{n}(A)=\bigcap_{i=1}^{m} \operatorname{ker} \tau_{1}^{c_{i, 1}} \cdots \tau_{n}^{c_{i, n}} .
$$

The group $\operatorname{Autgr}_{\mathrm{gr}} D_{n}$ of automorphisms of the algebraic group $D_{n}$ is naturally identified with $\mathrm{GL}_{n}(\mathbf{Z})$. Its natural action on the set of bases of the group $\mathrm{X}\left(D_{n}\right)$ is transitive. Therefore, there is an automorphism

$$
\varphi \in \operatorname{Aut}_{\mathrm{gr}} D_{n}
$$

such that $\tau_{i} \circ \varphi=\varepsilon_{i}$ for each $i$. From (15) it then follows that

$$
\varphi^{-1}\left(D_{n}(A)\right)=D_{n}(C), \quad C=\left(c_{i j}\right) \in \operatorname{Mat}_{m \times n}(\mathbf{Z}) .
$$

Since the map of varieties $D_{n} \rightarrow \mathbf{A}^{n},\left(t_{1} x_{1}, \ldots, t_{n} x_{n}\right) \mapsto\left(t_{1}, \ldots, t_{n}\right)$, is a birational isomorphism, by means of it we can identify the group $\mathrm{Cr}_{n}=$ Bir $\mathbf{A}^{n}$ with the group of birational automorphisms of the underlying variety of torus $D_{n}$. Then $\varphi$ becomes an element of the group $\mathrm{Cr}_{n}$ and from (16) and (17) it is easy to deduce that in this group we have the equality

$$
\varphi^{-1} D_{n}(A) \varphi=D_{n}(C) \text {. }
$$

Further, notice that if the base $\tau_{1}, \ldots, \tau_{n}$ is obtained from the base $\varepsilon_{1}, \ldots$ $\ldots, \varepsilon_{n}$ by an elementary transformation, then the matrix $C$ is obtained from $A$ by an elementary transformation of columns, and every elementary transformation of columns of $A$ is realizable in this way. Also, notice that if the 
sequence $\varphi_{1}, \ldots, \varphi_{m} \in \mathrm{X}\left(D_{n}\right)$ is obtained by an elementary transformation from the sequence $\lambda_{1}, \ldots, \lambda_{m}$, then $D_{n}(A)=\bigcap_{i=1}^{m} \operatorname{ker} \varphi_{i}$, the matrix $\left(c_{i j}\right)$ defined by the equalities $\varphi_{i}=\varepsilon_{1}^{c_{i, 1}} \cdots \varepsilon_{n}^{c_{i, n}}$ is obtained from $A$ by an elementary transformation of rows, and every elementary transformation of rows of $A$ is realizable in this way.

Clearly, the said implies the claim of lemma.

Corollary 1. If $S$ is the Smith normal form of matrix $A$, then the subgroups $D_{n}(A)$ and $D_{n}(S)$ are conjugate in $\mathrm{Cr}_{n}$.

\section{Lemma 2.}

(i) Let $q_{1} \leqslant \cdots \leqslant q_{r}$ be all the invariant factors of matrix $A \in \operatorname{Mat}_{m \times n}(\mathbf{Z})$. Then the group $D_{n}(A)$ is isomorphic to

$$
\boldsymbol{\mu}_{q_{1}} \times \cdots \times \boldsymbol{\mu}_{q_{r}} \times \mathbf{G}_{\mathrm{m}}^{n-r} .
$$

(ii) The closed $(n-m)$-dimensional subgroups of $D_{n}$ are every possible subgroups $D_{n}(A)$, where $A \in \operatorname{Mat}_{m \times n}(\mathbf{Z}), \operatorname{rk} A=m$, and only they.

(iii) $\mathcal{R}_{A}=\left\{\left(l_{1}, \ldots, l_{n}\right) \in \mathbf{Z}^{n} \mid D_{n}(A) \subseteq \operatorname{ker} \varepsilon_{1}^{l_{1}} \cdots \varepsilon_{n}^{l_{n}}\right\}$ for every $A \in$ $\operatorname{Mat}_{m \times n}(\mathbf{Z})$.

(iv) If $A \in \operatorname{Mat}_{s \times n}(\mathbf{Z}), B \in \operatorname{Mat}_{t \times n}(\mathbf{Z})$, then

(a) $D_{n}(A)=D_{n}(B)$ if and only if $\mathcal{R}_{A}=\mathcal{R}_{B}$;

(b) The following properties are equivalent:

$\left(\mathrm{b}_{1}\right) D_{n}(A)$ and $D_{n}(B)$ are conjugate in $\mathrm{GL}_{n}$;

$\left(\mathrm{b}_{2}\right) D_{n}(A)$ and $D_{n}(B)$ are conjugate in $N_{\mathrm{GL}_{n}}\left(D_{n}\right)$;

$\left(\mathrm{b}_{2}\right)$ by a permutation of columns, it is possible to tranform $B$ into a matrix $C$ such that $\mathcal{R}_{A}=\mathcal{R}_{C}$.

Proof. (i) Let $S=\left(s_{i j}\right)$ be the normal Smith form of matrix $A$. Then $s_{11}=$ $q_{1}, \ldots, s_{r r}=q_{r}$ and $s_{i j}=0$ in the other cases. Hence $D_{n}(S)$ is isomorphic to group (18). But $D_{n}(A)$ is isomorphic to $D_{n}(S)$ by Corollary 1. This proves (i).

(ii) From (i) we deduce that

$$
\operatorname{dim} D_{n}(A)=n-\operatorname{rk} A
$$

therefore, $\operatorname{dim} D_{n}(A)=n-m$, where rk $A=m$. Conversely, let $H$ be a closed subgroup of $D_{n}$ such that $\operatorname{dim} H=n-m$. Then $D_{n} / H$ is an $m$-dimensional torus [Bo91, p. 114] and therefore, there is an isomorphism $\alpha: D_{n} / H \rightarrow \mathbf{G}_{\mathrm{m}}^{m}$. Let $\lambda_{i} \in \mathrm{X}\left(D_{n}\right)$ be the composition of homomorphisms

$$
D_{n} \stackrel{\pi}{\longrightarrow} D_{n} / H \stackrel{\alpha}{\longrightarrow} \mathbf{G}_{\mathrm{m}}^{m} \stackrel{\mathrm{pr}_{i}}{\longrightarrow} \mathbf{G}_{\mathrm{m}}
$$

where $\pi \mathrm{s}$ the canonical projection and $\mathrm{pr}_{i}$ is the projection to the $i$ th factor. Then $H=\bigcap_{i=1}^{m}$ ker $\lambda_{i}$. From (12) we then deduce that $H=D_{n}(A)$ and from (19) that $\operatorname{rk} A=m$. This proves (ii).

(iii) From (11), (12) it follows that the left-hand side of the equality under proof is contained in the right-hand side. Proving the inverse inclusion consider a character $\lambda=\varepsilon_{1}^{l_{1}} \cdots \varepsilon_{n}^{l_{n}}$ whose kernel contains $D_{n}(A)$. Without changing $D_{n}(A)$ and $\mathcal{R}_{A}$, we can leave in $A$ only the rows that form a base of the group $\mathcal{R}_{A}$ removing the other rows, i.e., we can reduce our considerations 
to the case where rk $A=m$. Consider then the characters $\lambda_{1}, \ldots, \lambda_{m}$ defined by formula (12), and the homomorphism

$$
\varphi: D_{n} \rightarrow \mathbf{G}_{\mathrm{m}}^{m}, \quad g \mapsto\left(\lambda_{1}(g), \ldots, \lambda_{m}(g)\right) .
$$

In view of $(12)$, we have $\operatorname{ker} \varphi=D_{n}(A)$. From this and (19) it follows that $\operatorname{dim} \varphi\left(D_{n}\right)=m$. Therefore, $\varphi$ is a surjection. Hence $\mathbf{G}_{\mathrm{m}}^{m}$ is the quotient group of $D_{n}$ by $D_{n}(A)$ and $\varphi$ is the canonical homomorphism to it. Since $\lambda$ is constant on the fibers of $\varphi$, the universal property of quotient implies the existence of character $\mu: \mathbf{G}_{\mathrm{m}}^{m} \rightarrow \mathbf{G}_{\mathrm{m}}$ such that $\lambda=\mu \circ \varphi$. Hence $\lambda=$ $\lambda_{1}^{c_{1}} \cdots \lambda_{m}^{c_{m}}$ for some $c_{1}, \ldots, c_{m} \in \mathbf{Z}$, and this means that $\left(l_{1}, \ldots, l_{n}\right) \in \mathcal{R}_{A}$. This proves (iii).

(iv)(a) If $D_{n}(A)=D_{n}(B)$, then $\mathcal{R}_{A}=\mathcal{R}_{B}$ because of (iii). Conversely, if $\mathcal{R}_{A}=\mathcal{R}_{B}$, then $D_{n}(A)=D_{n}(B)$ because of (14). This proves (iv)(a).

(iv)(b) By fusion theorem [Se00, 1.1.1] the subgroups $D_{n}(A)$ and $D_{n}(B)$ are conjugate in $\mathrm{GL}_{n}$ if and only if they are conjugate in $N_{\mathrm{GL}_{n}}\left(D_{n}\right)$. But (8) and (12) imply that $D_{n}(A)$ and $D_{n}(B)$ are conjugate in $N_{\mathrm{GL}_{n}}\left(D_{n}\right)$ if and only if by a permutation of columns one can obtain from $B$ a matrix $C$ such that $D_{n}(A)=D_{n}(C)$. Because of (iii)(a), the latter equality is equivalent to the equality $\mathcal{R}_{C}=\mathcal{R}_{A}$. This proves (iv)(b).

\section{Corollary 2.}

(i) Let $\left(l_{1}, \ldots, l_{n}\right) \neq(0, \ldots, 0)$ and $d:=\operatorname{gcd}\left(l_{1}, \ldots, l_{n}\right)$. Then $D_{n}\left(l_{1}, \ldots, l_{n}\right)$ is isomorphic to $\boldsymbol{\mu}_{d} \times \mathbf{G}_{\mathrm{m}}^{n-1}$. In particular, the group $D_{n}\left(l_{1}, \ldots, l_{n}\right)$ is connected (i.e., is a torus) if and only if $d=1$.

(ii) The closed $(n-1)$-dimensional subgroups of $D_{n}$ are every possible subgroups $D_{n}\left(l_{1}, \ldots, l_{n}\right)$ with $\left(l_{1}, \ldots, l_{n}\right) \neq(0, \ldots, 0)$ and only they.

(iii) $D_{n}\left(l_{1}, \ldots, l_{n}\right)=D_{n}\left(l_{1}^{\prime}, \ldots, l_{n}^{\prime}\right)$ if and only if

$$
\left(l_{1}, \ldots, l_{n}\right)= \pm\left(l_{1}^{\prime}, \ldots, l_{n}^{\prime}\right) .
$$

(iv) The following properties are equivalent:

(ivi) $D_{n}\left(l_{1}, \ldots, l_{n}\right)$ and $D_{n}\left(l_{1}^{\prime}, \ldots, l_{n}^{\prime}\right)$ are conjugate in $\mathrm{GL}_{n}$;

(iv 2$) D_{n}\left(l_{1}, \ldots, l_{n}\right)$ and $D_{n}\left(l_{1}^{\prime}, \ldots, l_{n}^{\prime}\right)$ are conjugate in $N_{\mathrm{GL}_{n}}\left(D_{n}\right)$;

(iv3) there is a permutation $\sigma \in S_{n}$ such that

$$
\left(l_{1}, \ldots, l_{n}\right)= \pm\left(l_{\sigma(1)}^{\prime}, \ldots, l_{\sigma(n)}^{\prime}\right) .
$$

The following Lemma 3 gives an effective numerical criterion for the equality $\mathcal{R}_{A}=\mathcal{R}_{B}$ from Lemma 2(iii).

Let $A \in \operatorname{Mat}_{m \times n}(\mathbf{Z})$, rk $A=m$. For every strictly increasing sequence of $m$ integers $i_{1}, \ldots, i_{m}$ taken from the interval $[1, n]$, put

$$
p_{i_{1}, \ldots, i_{m}}(A):=\operatorname{det} A_{i_{1}, \ldots, i_{m}},
$$

where $A_{i_{1}, \ldots, i_{m}}$ is the submatrix of matrix $A$ obtained by intersecting rows with numbers $1, \ldots, m$ and columns with numbers $i_{1}, \ldots, i_{m}$ (it is natural to call the $p_{i_{1}, \ldots, i_{m}}(A)$ 's the Plücker coordinates of matrix $\left.A\right)$.

Lemma 3. For every two matrices $A$ and $B \in \operatorname{Mat}_{m \times n}(\mathbf{Z})$ of rank $m$ the following properties are equivalent:

(i) $\mathcal{R}_{A}=\mathcal{R}_{B}$. 
(ii) Two conditions hold:

(a) either $p_{i_{1}, \ldots, i_{m}}(A)=p_{i_{1}, \ldots, i_{m}}(B)$ for all $i_{1}, \ldots, i_{m}$, or $p_{i_{1}, \ldots, i_{m}}(A)=$ $-p_{i_{1}, \ldots, i_{m}}(B)$ for all $i_{1}, \ldots, i_{m}$

(b) for every sequence $i_{1}, \ldots, i_{m}$ such that $p_{i_{1}, \ldots, i_{m}}(A) \neq 0$, the following inclusion holds:

$$
B_{i_{1}, \ldots, i_{m}}\left(A_{i_{1}, \ldots, i_{m}}\right)^{-1} \in \operatorname{Mat}_{m \times m}(\mathbf{Z}) .
$$

Proof. Whereas rk $A=\operatorname{rk} B=m$, the rows of $A$ and $B$ form the bases in $\mathcal{R}_{A}$ and $\mathcal{R}_{B}$ respectively. Therefore, $\mathcal{R}_{A}=\mathcal{R}_{B}$ if and only if when there is a matrix $Q \in \mathrm{GL}_{m}(\mathbf{Z})$ such that

$$
A=Q B .
$$

Let $\mathcal{R}_{A}=\mathcal{R}_{B}$. Then (22) implies that

$$
A_{i_{1}, \ldots, i_{m}}=Q B_{i_{1}, \ldots, i_{m}}
$$

for every $i_{1}, \ldots, i_{m}$, and therefore, $p_{i_{1}, \ldots, i_{m}}(A)=\operatorname{det} Q p_{i_{1}, \ldots, i_{m}}(B)$ because of (20). Since $Q \in \mathrm{GL}_{m}(\mathbf{Z})$, we have $\operatorname{det} Q= \pm 1$. Hence condition (ii)(a) holds. If $p_{i_{1}, \ldots, i_{m}}(A) \neq 0$, then $p_{i_{1}, \ldots, i_{m}}(B) \neq 0$ as well, hence $B_{i_{1}, \ldots, i_{m}}$ is nondegenerate and $(23)$ implies that $Q=A_{i_{1}, \ldots, i_{m}}\left(B_{i_{1}, \ldots, i_{m}}\right)^{-1}$. Hence condition (ii)(b) holds. This proves (i) $\Rightarrow$ (ii).

Proving the inverse implication, consider $\mathbf{Z}^{n}$ as a subset of the coordinate vectors space (of rows) $\mathbf{Q}^{n}$. Condition (ii)(a) shows that the $\mathbf{Q}$-linear spans of subsets $\mathcal{R}_{A}$ and $\mathcal{R}_{B}$ of $\mathbf{Q}^{n}$ have the same Plücker coordinates. Hence these spans are one and the same linear subspace $L$ (see, e.g., [ShRe09, Theorem 10.1]). Since the rows of $A$ and rows of $B$ form two bases in $L$, there exists a matrix $P \in \mathrm{GL}_{m}(\mathbf{Q})$ such that $A=P B$. Therefore, $A_{i_{1}, \ldots, i_{m}}=P B_{i_{1}, \ldots, i_{m}}$ for every $i_{1}, \ldots, i_{m}$ and hence $P=A_{i_{1}, \ldots, i_{m}}\left(B_{i_{1}, \ldots, i_{m}}\right)^{-1}$ if $p_{i_{1}, \ldots, i_{m}}(B) \neq 0$. Then (ii)(b) implies that $P \in \mathrm{GL}_{n}(\mathbf{Z})$. Therefore, $\mathcal{R}_{A}=\mathcal{R}_{B}$. This proves (ii) $\Rightarrow($ i).

Remark 1.

1. In the proof of Lemma 3 it is established that, in fact, (ii)(a) implies that the matrix $B_{i_{1}, \ldots, i_{m}}\left(A_{i_{1}, \ldots, i_{m}}\right)^{-1}$ is independent of the choice of a sequence $i_{1}, \ldots, i_{m}$ for which $p_{i_{1}, \ldots, i_{m}}(A) \neq 0$. Therefore, (ii)(b) follows from (ii)(a) and feasibility of (21) for any one such a sequence.

2. If $m=1$, then (ii)(b) follows from (ii)(a) (but for $m>1$ this is not so).

Theorem 1 (Classificaton of diagonalizable subgroups of Aff $n$ up to conjugacy in $\mathrm{Cr}_{n}$ ).

(i) Two diagonalizable subgroups of the group $\mathrm{Aff}_{n}$ are conjugate in $\mathrm{Cr}_{n}$ if and only if they are isomorphic.

(ii) Any diagonalizable subgroup $G$ of the group $\mathrm{Aff}_{n}$ is conjugate in $\mathrm{Cr}_{n}$ to a unique closed subgroup of the torus $D_{n}$ that has the form

$$
\operatorname{ker} \varepsilon_{r+1}^{d_{1}} \cap \ldots \cap \operatorname{ker} \varepsilon_{r+s}^{d_{s}} \cap \operatorname{ker} \varepsilon_{r+s+1} \cap \ldots \cap \operatorname{ker} \varepsilon_{n}
$$

where $0 \leqslant r \leqslant n, 0 \leqslant s \leqslant n, r+s \leqslant n, 2 \leqslant d_{1}$ and $d_{i}$ divides $d_{i+1}$ for every $i<s$. The integers determining subgroup (24) have the following meaning: $r=\operatorname{dim} G$ and $d_{1}, \ldots, d_{s}$ are all the invariant factors of the finite Abelian group $G / G^{0}$. 
Proof. Since maximal reductive subgroups of algebraic group are conjugate (see [BoSe64, 5.1]) and $\mathrm{GL}_{n}$ is one of them in $\mathrm{Aff}_{n}$, every diagonalizable subgroup of $\mathrm{Aff}_{n}$ is conjugate to a subgroup of $\mathrm{GL}_{n}$. In turn, every diagonalizable subgroup of $\mathrm{GL}_{n}$ is conjugate to a subgroup of the torus $D_{n}$ (see [Bo91, I.4.6]). In view of Lemma 2(ii) this shows that it suffices to prove (i) for the subgroups $D_{n}(A)$ and $D_{n}(B)$ of the torus $D_{n}$. Further, appending, if necessary, zero rows we may assume that $A$ and $B$ have the same number of rows. Let now $D_{n}(A)$ and $D_{n}(B)$ be isomorphic. Then their dimensions are equal and the groups $D_{n}(A) / D_{n}(A)^{0}$ and $D_{n}(B) / D_{n}(B)^{0}$ have the same invariant factors. This and Lemma 2(i) imply that the matrices $A$ and $B$ have the same invariant factors (because the latter are obtained by appending the same number of 1's to the invariant factors of the specified groups). Hence the normal Smith forms of $A$ and $B$ are equal. By Corollary 1 this implies that $D_{n}(A)$ and $D_{n}(B)$ are conjugate in $\mathrm{Cr}_{n}$. This proves (i).

It is clear that the integers determining subgroup (24) have the meaning specified in (ii). Since every diagonalizable group is a direct product of a finite Abelian group and a torus, it is uniquely, up to isomorphism, determined by its dimension and the invariant factors of the group of connected components. This and (i) implies (ii).

Corollary 3. $D_{n}(A)$ and $D_{n}(B)$ are conjugate in $\mathrm{Cr}_{n}$ if and only if $A$ and $B$ have the same invariant factors.

Corollary 4. Every torus $T$ in $\mathrm{Aff}_{n}$ is conjugate in $\mathrm{Cr}_{n}$ to the torus $D_{r}$, $r=\operatorname{dim} T$.

Proof. This follows from Theorem 1(ii).

Corollary 5. Every two isomorphic finite Abelian subgroups of $\mathrm{Aff}_{n}$ are conjugate in $\mathrm{Cr}_{n}$.

Proof. Since char $k=0$, every element in Aff $_{n}$ of finite order is semisimple. Hence every finite Abelian subgroup of Aff $_{n}$ is reductive, and therefore, is conjugate in $\mathrm{Aff}_{n}$ to a subgroups of $\mathrm{GL}_{n}$ (see the proof of Theorem 1). But every commutative subgroup of $\mathrm{GL}_{n}$ consisting only of semisimple elements is diagonalizable (see [Bo91, Prop.4.6(b)]). The claim now follows from Theorem 1(i).

Corollary 6 ([Bl06 ${ }_{1}$, Thm.1]). Every two elements in Aff ${ }_{n}$ of the same finite order are conjugate in $\mathrm{Cr}_{n}$.

\section{TORI IN $\mathrm{Cr}_{n}$, Aut $\mathbf{A}^{n}$, AND Aut* $\mathbf{A}^{n}$}

Theorem 2 (Tori in $\mathrm{Cr}_{n}$ ).

(i) In $\mathrm{Cr}_{n}$ there are no tori of dimension $>n$.

(ii) In $\mathrm{Cr}_{n}$ every $r$-dimensional torus for $r=n, n-1, n-2$ is conjugate to the torus $D_{r}$.

(iii) If $n \geqslant 5$, then in $\mathrm{Cr}_{n}$ there are $(n-3)$-dimensional tori that are not conjugate to subtori of the torus $D_{n}$.

(iv) Every r-dimensional torus in $\mathrm{Cr}_{n}$ is conjugate in $\mathrm{Cr}_{n+r}$ to the torus $D_{r}$.

(v) In $\mathrm{Cr}_{\infty}$ every -dimensional torus is conjugate to the torus $D_{r}$. 
Proof. (i) This is proved, e.g., in [De70], see also [Po12, Cor. 2.2].

(ii) According to [B-B66, Cor.2] (see also [Po12, Cor.2.4(b)]), every rdimensional torus in $\mathrm{Cr}_{n}$ for $r=n, n-1, n-2$ is conjugate to a subtorus of the torus $D_{n}$. Therefore, the claim follows from Corollary 4.

(iii) This is proved in [Po12, Cor. 2.5].

(iv) According to [Po12, Thm. 2.6], every $r$-dimensional torus in $\mathrm{Cr}_{r}$ is conjugate in $\mathrm{Cr}_{n+r}$ to a subtorus of the torus $D_{n+r}$. Therefore, the claim follows from Corollary 4.

(v) This follows from (iv).

\section{Corollary 7.}

(i) In $\mathrm{Cr}_{n}$ every $n$-dimensional torus is maximal.

(ii) In $\mathrm{Cr}_{n}$ there are no maximal $(n-1)$ - and $(n-2)$-dimensional tori.

(iii) For $n \geqslant 5$, in $\mathrm{Cr}_{n}$ there are maximal $(n-3)$-dimensional tori.

Remark 2. For $n \leqslant 3$, Theorem 2 gives the classification of tori in $\mathrm{Cr}_{n}$ up to conjugacy: the classes of conjugate nontrivial tori are exhausted by that of the tori $D_{1}, \ldots, D_{n}$.

Let $\left(l_{1}, \ldots, l_{n}\right) \in \mathbf{Z}^{n}$ be a nonzero element with $\operatorname{gcd}\left(l_{1}, \ldots, l_{n}\right)=1$. Clearly, the homomorphism

$$
\mathbf{G}_{\mathrm{m}} \rightarrow D_{n}, \quad t \mapsto\left(t^{l_{1}} x_{1}, \ldots, t^{l_{n}} x_{n}\right),
$$

is an embedding and every embedding $\mathbf{G}_{\mathrm{m}} \hookrightarrow D_{n}$ is of this form. Denote by $T\left(l_{1}, \ldots, l_{n}\right)$ the image of embedding $(25)$. It is a one-dimensional torus in $D_{n}$ and every one-dimensional torus in $D_{n}$ is of this form.

Lemma 4. The following properties are equivalent:

(i) $T\left(l_{1}, \ldots, l_{n}\right)=T\left(l_{1}^{\prime}, \ldots, l_{n}^{\prime}\right)$.

(ii) $\left(l_{1}, \ldots, l_{n}\right)= \pm\left(l_{1}^{\prime}, \ldots, l_{n}^{\prime}\right)$.

Proof. (i) $\Rightarrow$ (ii) This is clear.

(ii) $\Rightarrow$ (i) Assume that (i) holds. Take an element $t \in \mathbf{G}_{\mathrm{m}}$ of infinite order. From (i) and the definition of $T\left(l_{1}, \ldots, l_{n}\right)$ we deduce that there is an element $s \in \mathbf{G}_{\mathrm{m}}$ such that $t^{l_{i}}=s^{l_{i}^{\prime}}$ for every $i=1, \ldots, n$. Hence $t^{l_{i} l_{j}^{\prime}}=s^{l_{i}^{\prime} l_{j}^{\prime}}=t^{l_{j} l_{i}^{\prime}}$ for every two nonequal integers $i$ and $j$ taken from the interval $[1, n]$. Since the order of $t$ is infinite, this implies that $l_{i} l_{j}^{\prime}-l_{j} l_{i}^{\prime}=0$. Hence

$$
\operatorname{rk}\left(\begin{array}{lll}
l_{1} & \ldots & l_{n} \\
l_{1}^{\prime} & \ldots & l_{n}^{\prime}
\end{array}\right)=1 .
$$

Therefore, $\left(l_{1}, \ldots, l_{n}\right)=\gamma\left(l_{1}^{\prime}, \ldots, l_{n}^{\prime}\right)$ for some $\gamma \in \mathbf{Q}$, or, equivalently, $p\left(l_{1}, \ldots, l_{n}\right)=q\left(l_{1}^{\prime}, \ldots, l_{n}^{\prime}\right)$, where $p, q \in \mathbf{Z}, \operatorname{gcd}(p, q)=1$. Hence $p$ divides each of $l_{1}^{\prime}, \ldots, l_{n}^{\prime}$, and $q$ divides each of $l_{1}, \ldots, l_{n}$. Since the integers in each of these two sets are coprime, this implies that $\gamma= \pm 1$, i.e., (ii) holds.

Theorem 3 (Tori in Aut $\mathbf{A}^{n}$ ).

(i) In Aut $\mathbf{A}^{n}$ every $n$-dimensional torus is conjugate to the torus $D_{n}$. 
(ii) In Aut $\mathbf{A}^{n}$ all $(n-1)$-dimensional tori are exhausted, up to conjugacy in Aut $\mathbf{A}^{n}$, by all the groups of the form $D_{n}\left(l_{1}, \ldots, l_{n}\right)$ with $\left(l_{1}, \ldots, l_{n}\right) \neq(0, \ldots, 0)$ and $\operatorname{gcd}\left(l_{1}, \ldots, l_{n}\right)=1$.

(iii) In Aut $\mathbf{A}^{3}$ all one-dimensional tori are exhaused, up to conjugacy, by all the groups of the form $T\left(l_{1}, l_{2}, l_{3}\right)$.

Proof. According to [B-B66] and [B-B67], every $r$-dimensional torus in the group Aut $\mathbf{A}^{n}$ for $r=n$ and $n-1$, respectively, is conjugate to a subtorus of the torus $D_{n}$. This and Corollary 2 imply (i) and (ii).

According to [KKM-LR97], every one-dimensional torus in Aut $\mathbf{A}^{3}$ is conjugate to subtorus of the torus $D_{3}$. This implies (iii).

Theorem 4 (Tori in Aut $^{*} \mathbf{A}^{n}$ ).

(i) In $\mathrm{Aut}^{*} \mathbf{A}^{n}$ there are no tori of dimension $>n-1$.

(ii) In $\mathrm{Aut}^{*} \mathbf{A}^{n}$ every $(n-1)$-dimensional torus is maximal and conjugate to the torus $D_{n}^{*}($ see $(9))$.

Proof. (i) If Aut* $\mathbf{A}^{n}$ contains an $n$-dimensional torus $T$, then by Theorem 3 (i) there is an element $g \in$ Aut $\mathbf{A}^{n}$ such that

$$
T=g D_{n} g^{-1} \text {. }
$$

Replacing $g$ by $g z$, where $z \in D_{n}$ is an element such that $\operatorname{Jac}(z)=\operatorname{det} z=$ $1 / \operatorname{Jac}(g)$, we may assume that $g \in \operatorname{Aut}^{*} \mathbf{A}^{n}$. This and (26) imply that $D_{n} \subset$ Aut $^{*} \mathbf{A}^{n},-$ a contradiction. This proves (i).

(ii) Let $S$ be an $(n-1)$-dimensional torus in Aut* $\mathbf{A}^{n}$. By Theorem 3(i) and Corollary (ii) of Lemma 2 , there are $g \in$ Aut $\mathbf{A}^{n}$ and $\left(l_{1}, \ldots, l_{n}\right) \in \mathbf{Z}^{n}$ such that

$$
S=g D_{n}\left(l_{1}, \ldots, l_{n}\right) g^{-1} .
$$

As in the proof of (i), we may assume that $g \in \operatorname{Aut}^{*} \mathbf{A}^{n}$. From (27) it then follows that $D_{n}\left(l_{1}, \ldots, l_{n}\right) \subset$ Aut $^{*} \mathbf{A}^{n}$. This and $D_{n} \cap$ ker Jac $=D_{n}^{*}:=$ $D_{n}(1, \ldots, 1)$ imply the inclusion $D_{n}\left(l_{1}, \ldots, l_{n}\right) \subseteq D_{n}^{*}$. By Corollary (i) of Lemma 2 , both sides of this inclusion are $(n-1)$-dimensional tori. Hence it is the equality. This proves (ii)

Remark 3. For Aut $\mathbf{A}^{n}$ and $\mathrm{Aut}^{*} \mathbf{A}^{n}$, unlike for $\mathrm{Cr}_{n}$ (see Corollary $7(\mathrm{iii})$ ), at present nothing is known on the existence of tori of nonmaximal dimension. This problem is intimately related to the Cancellation Problem: Is there an affine variety $X$ not isomorphic to $\mathbf{A}^{m}, m=\operatorname{dim} X$, such that $X \times \mathbf{A}^{d}$ is isomorphic to $\mathbf{A}^{m+d}$ for some $d$ ? If the answer is positive, then in Aut $\mathbf{A}^{n}$ and Aut* $\mathbf{A}^{n}$ with $n=m+d$ there exists a maximal torus $T$ of a nonmaximal dimension. Indeed, multiplying, if necessary, $X \times \mathbf{A}^{d}$ by $\mathbf{A}^{1}$, one may assume that $d \geqslant 2$. Let $\lambda$ be the character $t \mapsto t$ of the torus $\mathbf{G}_{\mathrm{m}}$. Consider the linear action of $\mathbf{G}_{\mathrm{m}}$ on $\mathbf{A}^{d}$ with precisely two isotypic components: one is $(d-1)$-dimensional of type $\lambda$, another is one-dimensional of typer $\lambda^{1-d}$. It determines the action of $\mathbf{G}_{\mathrm{m}}$ on $X \times \mathbf{A}^{d}$ via the second factor and hence, an action of $\mathbf{G}_{\mathrm{m}}$ on $\mathbf{A}^{n}$. Consider in Aut $\mathbf{A}^{n}$ the torus that is the image of $\mathbf{G}_{\mathrm{m}}$ under the homomorphism determined by this action. The construction implies that this torus lies in Aut* $\mathbf{A}^{n}$. Let $T$ (respectively, $T^{\prime}$ ) be a maximal torus of Aut $\mathbf{A}^{n}$ (respectively, of Aut $^{*} \mathbf{A}^{n}$ ) that contains this image. If $T$ is 
$n$-dimensional (respectively, $T^{\prime}$ is $(n-1)$-dimensional), then by Theorem 3(i) (respectively, by Theorem 4) it is conjugate to a subtorus of the torus $D_{n}$ and, therefore, the specified action of $T$ (respectively, $T^{\prime}$ ) on $\mathbf{A}^{n}$ is equivalent to a linear one. Hence the action of $\mathbf{G}_{\mathrm{m}}$ on $\mathbf{A}^{n}$ is equivalent to a linear one as well, and therefore, the set $F$ of its fixed points is isomorphic to an affine space. But, by the construction, in $\mathbf{A}^{d}$ there is a single $\mathbf{G}_{\mathrm{m}}$-fixed point, consequently, $F$ is isomorphic to $X-$ a contradiction. $^{2}$

\section{Orbits and stabilizers of the action of $D_{n}\left(l_{1}, \ldots, l_{n}\right)$ on $\mathbf{A}^{n}$}

Here we establish some properties, needed for what follows, of orbits and stabilizers of the natural action on $\mathbf{A}^{n}$ of the group

$$
G:=D_{n}\left(l_{1}, \ldots, l_{n}\right) \text {. }
$$

Clearly, every coordinate hyperplane

$$
H_{i}:=\left\{a \in \mathbf{A}^{n} \mid x_{i}(a)=0\right\}
$$

is $G$-invariant.

Lemma 5. The G-stabilizer of every point of $\mathbf{A}^{n} \backslash \bigcup_{i=1}^{n} H_{i}$ is trivial.

(i) If $\left(l_{1}, \ldots, l_{n}\right)=(0, \ldots, 0)$, then $\mathbf{A}^{n} \backslash \bigcup_{i=1}^{n} H_{i}$ is a $G$-orbit, and dimension of the $G$-stabilizer of every point of $\bigcup_{i=1}^{n} H_{i}$ is positive.

(ii) If $\left(l_{1}, \ldots, l_{n}\right) \neq(0, \ldots, 0)$, then $\operatorname{dim} G \cdot a=n-1$ for every point $a \in \mathbf{A}^{n} \backslash \bigcup_{i=1}^{n} H_{i}$.

Proof. This immediately follows from (28), (12), (4) and Lemma 2.

We now consider the case

$$
\left(l_{1}, \ldots, l_{n}\right) \neq(0, \ldots, 0) .
$$

Lemma 6. If $l_{i} \neq 0$, then the open subsubset $\mathcal{O}_{i}:=H_{i} \backslash \bigcup_{j \neq i} H_{j}$ in $H_{i}$ is a G-orbit.

Proof. By virtue of $G$-invariance of $\mathcal{O}_{i}$, it suffices to show that $\mathcal{O}_{i}$ is contained in a $G$-orbit. Since $l_{i} \neq 0$, the equation $x^{l_{i}}=\alpha$ has a solution for any $\alpha \in k, \alpha \neq 0$. This and (28), (12) imply that for any two points

$b=\left(b_{1}, \ldots, b_{i-1}, 0, b_{i+1}, \ldots, b_{n}\right), c=\left(c_{1}, \ldots, c_{i-1}, 0, c_{i+1}, \ldots, c_{n}\right) \in H_{i} \backslash \bigcup_{j \neq i} H_{j}$

there exists an element $g=\left(t_{1} x_{1}, \ldots, t_{n} x_{n}\right) \in G$ such that $t_{j}=b_{j}^{-1} c_{j}$ for every $j \neq i$. From (4) we then obtain that $g \cdot b=c$, as required.

Lemma 7. The following properties are equivalent:

\footnotetext{
${ }^{2}$ Added on August 2, 2012. Theorems 3 and 4 and Remark 3 remain valid when the characteristic $p$ of the ground field $k$ is positive. In the preprint N. Gupta, A counter-example to the Cancellation Problem for the affine space $\mathbf{A}^{3}$ in characteristic $p$, arXiv:1208.0483 published today, is proved that the hypersurface $X$ in $\mathbf{A}^{4}$ defined by the equation $x_{1}^{m} x_{2}+$ $x_{3}^{p^{e}}+x_{4}+x^{s p}=0$, where $m, e, s$ are positive integers such that $p^{e} \nmid s p$ and $s p \nmid p^{e}$, is not isomorphic to $\mathbf{A}^{3}$, but $X \times \mathbf{A}^{1}$ is isomorphic to $\mathbf{A}^{4}$. By Remark 3, this implies that, for char $k>0$ and every integer $n \geqslant 5$, there are maximal tori of nonmaximal dimension in Aut $\mathbf{A}^{n}$ and Aut $^{*} \mathbf{A}^{n}$.
} 
(i) all the numbers $l_{1}, \ldots, l_{n}$ are nonzero and have the same sign;

(ii) G-orbit of every point of $\mathbf{A}^{n} \backslash \bigcup_{i=1}^{n} H_{i}$ is closed in $\mathbf{A}^{n}$;

(iii) G-orbit of some point of $\mathbf{A}^{n} \backslash \bigcup_{i=1}^{n} H_{i}$ is closed in $\mathbf{A}^{n}$.

Proof. Consider a point

$$
a=\left(a_{1}, \ldots, a_{n}\right) \in \mathbf{A}^{n} \backslash \bigcup_{i=1}^{n} H_{i} .
$$

Assume that (i) holds. Suppose that the $G$-orbit of the point $a$ is not closed in $\mathbf{A}^{n}$. Then its boundary can be accessed by a one-parameter subgroup, i.e., there is homomorphism $\varphi: \mathbf{G}_{\mathrm{m}} \rightarrow G$ such that there is a limit

$$
\lim _{t \rightarrow 0} \varphi(t) \cdot a=b \in \overline{G \cdot a} \backslash G \cdot a
$$

(see [PoVi94, Thm. 6.9]); the latter means that the morphism $\mathbf{G}_{\mathrm{m}}=\mathbf{A}^{1} \backslash$ $\{0\} \rightarrow \mathbf{A}^{n}, t \mapsto \varphi(t) \cdot a$, extends to a morphism $\mathbf{A}^{1} \rightarrow \mathbf{A}^{n}$ that maps 0 to the point $b$. As $\varphi$ is algebraic, there is a vector $\left(d_{1}, \ldots, d_{n}\right) \in \mathbf{Z}^{n}$ such that $\varphi(t)=\left(t^{d_{1}} x_{1}, \ldots, t^{d_{n}} x_{n}\right)$ for every $t \in \mathbf{G}_{\mathrm{m}}$. Since $\varphi(t) \cdot a=\left(t^{d_{1}} a_{1}, \ldots, t^{d_{n}} a_{n}\right)$ and $a_{i} \neq 0$ for every $i$, the existence of the specified limit means that

$$
d_{1} \geqslant 0, \ldots, d_{n} \geqslant 0 \text {. }
$$

On the other hand, it follows from $\varphi(t) \in G$ and (28), (12) that $t^{d_{1} l_{1}+\cdots+d_{n} l_{n}}$ $=1$ for every $t$, i.e.,

$$
d_{1} l_{1}+\cdots+d_{n} l_{n}=0 .
$$

But (31), (32) and condition (i) imply that $d_{1}=\cdots=d_{n}=0$. Hence $b=a$ contrary to (30). This contradiction proves (i) $\Rightarrow$ (ii).

Conversely, assume that (i) is not fulfilled, i.e., among $l_{1}, \ldots, l_{n}$ there are either two nonzero numbers with different signs, or one number equal to zero. In the first case, let, for instance, be $l_{1}>0, l_{2}<0$. Then (12) implies that the image of the homomorphism $\varphi: \mathbf{G}_{\mathrm{m}} \rightarrow D_{n}, t \mapsto$ $\left(t^{-l_{2}} x_{1}, t^{l_{1}} x_{2}, x_{3}, \ldots, x_{n}\right)$, lies in $G$. Since

$$
\lim _{t \rightarrow 0} \varphi(t) \cdot a=\left(0,0, a_{3}, \ldots, a_{n}\right) \notin G \cdot a,
$$

this shows that the orbit $G \cdot a$ is not closed. In the second case, let, say, $a_{1}=0$. Then $G$ contains the image of the homomorphism $\varphi: \mathbf{G}_{\mathrm{m}} \rightarrow D_{n}$, $t \mapsto\left(t x_{1}, x_{2}, \ldots, x_{n}\right)$ and, since

$$
\lim _{t \rightarrow 0} \varphi(t) \cdot a=\left(0, a_{2}, \ldots, a_{n}\right) \notin G \cdot a,
$$

the orbit $G \cdot a$ is not closed. This proves (iii) $\Rightarrow(\mathrm{i})$.

From Lemmas 5, 6, and 7 we infer

Corollary 8. If all the numbers $l_{1}, \ldots, l_{n}$ are nonzero and have the same sign, then in $\mathbf{A}^{n}$ there are precisely $n$ nonclosed $(n-1)$-dimensional $G$ orbits - these are the orbits $\mathcal{O}_{1}, \ldots, \mathcal{O}_{n}$ from Lemma 6.

Remark 4. Recall from [Po70] that an action of an algebraic group on an algebraic variety is called stable, if orbits of points in general position are closed. Lemma 7 shows that the following properties are equivalent:

(i) all the numbers $l_{1}, \ldots, l_{n}$ are nonzero and have the same sign;

(ii) the action $G$ on $\mathbf{A}^{n}$ is stable. 
Lemma 8. Assume that none of the numbers $l_{1}, \ldots, l_{n}$ are equal to \pm 1 . Then the following properties of the point $a=\left(a_{1}, \ldots, a_{n}\right) \in \mathbf{A}^{n}$ are equivalent:

(i) a has a nontrivial G-stabilizer;

(ii) $a \in \bigcup_{i=1}^{n} H_{i}$.

Proof. Lemma 5 implies (i) $\Rightarrow$ (ii). Now assume that (ii) holds. Then there are the indices $i_{1}, \ldots, i_{s}$, where $s \geqslant 1$, such that $a_{j}=0$ for $j=i_{1}, \ldots, i_{s}$ and $a_{j} \neq 0$ for other $j$ 's. It follows from (4), (28), and (12) that an element $\left(t_{1} x_{1}, \ldots, t_{n} x_{n}\right) \in D_{n}$ lies in the $G$-stabilizer of the point $a$ if and only if $t_{j}=1$ for $j \neq i_{1}, \ldots, i_{s}$ and

$$
t_{i_{1}}^{l_{i_{1}}} \cdots t_{i_{s}}^{l_{i_{s}}}=1 .
$$

Since there is no \pm 1 among the numbers $l_{1}, \ldots, l_{n}$ and $k$ is an algebraically closed field of characteristic zero, equality (33), considered as the equation in $t_{i_{1}}, \ldots, t_{i_{s}}$, has at least two solutions. Hence the $G$-stabilizer of the point $a$ is nontrivial. This proves (ii) $\Rightarrow(\mathrm{i})$.

Lemma 9. If among $l_{1}, \ldots, l_{n}$ there are two nonzero numbers with different signs, then every $G$-orbit contains $(0, \ldots, 0)$ in its closure.

Proof. For definiteness, let, for instance, be

$$
l_{1}>0, l_{2} \geqslant 0, \ldots, l_{s} \geqslant 0, l_{s+1}<0, l_{s+2} \leqslant 0, \ldots, l_{n} \leqslant 0 .
$$

Choose a positive integer $d$ so large that

$$
q:=l_{2}+\cdots+l_{s}+d l_{s+1}+l_{s+2}+\cdots+l_{n}<0 .
$$

Since $-q l_{1}+l_{1} l_{2}+\cdots+l_{1} l_{s}+l_{1} d l_{s+1}+l_{1} l_{s+2}+\ldots+l_{1} l_{n}=0$, from (28) and (12) we infer that the image of the homomorphism

$$
\varphi: \mathbf{G}_{\mathrm{m}} \rightarrow D_{n}, \quad t \mapsto\left(t^{-q} x_{1}, t^{l_{1}} x_{2}, \ldots, t^{l_{1}} x_{s}, t^{l_{1} d} x_{s+1}, t^{l_{1}} x_{s+2} \ldots, t^{l_{1}} x_{n}\right)
$$

lies in $G$. On the other hand, since the numbers $-q, l_{1}$ and $d$ are positive, (4) implies that for every point $a \in \mathbf{A}^{n}$ the $\operatorname{limit}_{t \rightarrow 0} \lim _{t \rightarrow}(t) \cdot a$ exists and is equal to $(0, \ldots, 0)$.

Now consider the case where 0 and \pm 1 are contained among $l_{1}, \ldots, l_{n}$, there are at least two nonzero $l_{i}$ 's, and all of them have the same sign. By (12), without loss of generality we may assume that this sign is positive. Up to replacing the group $G$ by its conjugate by means of an element of $N_{\mathrm{GL}_{n}}\left(D_{n}\right)$, we may then assume that

$$
\begin{gathered}
l_{1}=\ldots=l_{p}=1, l_{p+1} \geqslant 2, \ldots, l_{q} \geqslant 2, l_{q+1}=\ldots=l_{n}=0, \\
\text { where } p \geqslant 1, n>q \geqslant p \text { and } q \geqslant 2 .
\end{gathered}
$$

Lemma 10. Assume that (35) holds. Take a point $a=\left(a_{1}, \ldots, a_{n}\right) \in \mathbf{A}^{n}$.

(i) Let $a \notin \bigcup_{i=1}^{n} H_{i}$. Then the orbit $G \cdot b$, where $b=\left(a_{1}, \ldots, a_{q}, 0, \ldots, 0\right)$, lies in the closure of the orbit $G \cdot a$, is closed, and $\operatorname{dim} G \cdot b=q-1$.

(ii) Let $a \in H_{i}$. Then the group $G_{a}$ is

(a) trivial if $1 \leqslant i \leqslant p$ and $a \in \mathcal{O}_{i}$ (see Lemma 6$)$;

(b) nontrivial and finite if $p+1 \leqslant i \leqslant q$ and $a \in \mathcal{O}_{i}$; 
(c) has positive dimension if $i>q$.

Proof. From (28), (12), and (35) it follows that the image of the homomorphism

$$
\varphi: \mathbf{G}_{\mathrm{m}} \rightarrow D_{n}, \quad t \mapsto\left(x_{1}, \ldots, x_{q}, t x_{q+1}, \ldots, t x_{n}\right),
$$

lies in $G$. Hence the point

$$
\lim _{t \rightarrow 0} \varphi(t) \cdot a=\left(a_{1}, \ldots, a_{q}, 0, \ldots, 0\right)=b
$$

lies in the closure of the orbit $G \cdot a$.

Now let $a \notin \bigcup_{i=1}^{n} H_{i}$. If the orbit $G \cdot b$ is not closed, then, as in the proof of Lemma 7 , there exist a homomorphism

$$
\psi: \mathbf{G}_{\mathrm{m}} \rightarrow G, \quad t \mapsto\left(t^{d_{1}} x_{1}, \ldots, t^{d_{n}} x_{n}\right),
$$

such that $c:=\lim _{t \rightarrow 0} \psi(t) \cdot b \in \overline{G \cdot b} \backslash G \cdot b$. From (36) it follows that $d_{1} \geqslant$ $0, \ldots, d_{q} \geqslant 0$, and from (35), (28), and (12) it follows that $d_{1} l_{1}+\cdots+d_{q} l_{q}=0$. Since $l_{1}, \ldots, l_{q}$ are positive, this yields $d_{1}=\ldots=d_{q}=0$. In view of (36), from this we infer that $\psi(t) \cdot b=b$ for every $t$, and therefore, $c=b-\mathrm{a}$ contradiction. Thus, $G \cdot b$ is closed. Since $a_{1}, \ldots, a_{q}$ are nonzero, (28), (12), (4), (35), and (36) imply that an element $\left(t_{1} x_{1}, \ldots, t_{n} x_{n}\right) \in D_{n}$ lies in $G_{b}$ if and only if $t_{1}=\ldots=t_{q}=1$. This proves (i).

The arguments analogous to that used in the proof of Lemma 8 yield (ii).

Finally, consider the case where one of the numbers $l_{1}, \ldots, l_{n}$ is equal to \pm 1 (in view of (12), without loss of generality we may assume that it is equal to 1 ), and all the others are equal to 0.

Lemma 11. Let $l_{i}=1$ and let $l_{j}=0$ for $j \neq i$. For any $s \in k$ denote by $H(s)$ the hyperplane in $\mathbf{A}^{n}$ defined by the equation $x_{i}+s=0$. Then:

(i) $\bigcup_{j \neq i} H_{j}$ is the set of points with nontrivial G-stabilizer (that automatically has positive dimension).

(ii) The open subset $H(s) \backslash \bigcup_{j \neq i} H_{j}$ of $H(s)$ is an $(n-1)$-dimensional $G$-orbit and every $(n-1)$-dimensional $G$-orbit is of this form.

Proof. Part (i) immediately follows from (4), (28), and (12), and part (ii) follows from (i), the invariance of $H(\alpha)$, and the equality $\operatorname{dim} G=\operatorname{dim} H(\alpha)=$ $n-1$.

\section{The group $N_{\text {Aut }} \mathbf{A}^{n}\left(D_{n}\left(l_{1}, \ldots, l_{n}\right)\right)$}

First, we shall prove several general statements about normalizers for the actions on arbitrary affine varieties.

Lemma 12. Let $X$ be an irreducible affine variety and let $G$ be an algebraic subgroup of Aut $X$. Then the following properties are equivalent:

(i) $N_{\text {Aut } X}(G)$ is an algebraic subgroup of Aut $X$.

(ii) The natural action of $N_{\text {Aut } X}(G)$ on $k[X]$ is locally finite. 
Proof. (i) $\Rightarrow$ (ii) This follows from the fact that the natural action on $k[X]$ of every algebraic subgroup of Aut $X$ is locally finite (see [Bo91, Prop. 1.9]).

(ii) $\Rightarrow$ (i) Assume that (ii) holds. Then in $k[X]$ there is an $N_{\text {Aut } X}(G)$ invariant finite dimensional $k$-linear subspace $V$ containing a system of generators of the $k$-algebra $k[X]$. Hence the homomorphism

$$
\rho: N_{\mathrm{Aut} X}(G) \rightarrow \mathrm{GL}\left(V^{*}\right)
$$

determined by the action of $N_{\text {Aut } X}(G)$ on $V$ is an embedding. Consider the $N_{\text {Aut } X}(G)$-equivariant map

$$
\iota: X \rightarrow V^{*}, \quad \iota(x)(f):=f(x) \quad \text { for every } x \in X, f \in V .
$$

The standard argument (see[Bo91, Prop. 1.12]) shows that $\left.\rho\right|_{G}$ is a morphism of algebraic groups, and $\iota$ is a closed embedding. Identify $X$ with $\iota(X)$ by means of $\iota$, and $N_{\text {Aut } X}(G)$ with $\rho\left(N_{\text {Aut } X}(G)\right)$ by means of $\rho$. Then $X$ is a closed subvariety of $V^{*}$, and $N_{\text {Aut } X}(G)$ and $G$ are the subgroups of $\mathrm{GL}\left(V^{*}\right)$; besides, $G$ is closed, and

$$
\begin{gathered}
N_{\text {Aut } X}(G) \subset N_{\mathrm{GL}\left(V^{*}\right)}(G) \cap \operatorname{Tran}_{\mathrm{GL}\left(V^{*}\right)}(X, X), \quad \text { where } \\
\operatorname{Tran}_{\mathrm{GL}\left(V^{*}\right)}(X, X):=\left\{g \in \operatorname{GL}\left(V^{*}\right) \mid g \cdot X \subset X\right\} .
\end{gathered}
$$

In fact, in the right-hand side of (39) the equality $g \cdot X=X$ automatically holds: indeed, $X$ is irreducible and closed in $V^{*}$, and $g \in$ Aut $V^{*}$ implies that $g \cdot X$ is a closed subset of $X$ of the same dimension as $X$. Hence $\operatorname{Tran}_{\mathrm{GL}\left(V^{*}\right)}(X, X)$, as well as $N_{\mathrm{GL}\left(V^{*}\right)}(G)$, is a subgroup of $\mathrm{GL}\left(V^{*}\right)$, and therefore, the right-hand side of (38) is a subgroup of $\mathrm{GL}\left(V^{*}\right)$. Its elements normalize $G$ an, being restricted to $X$, are the automorphisms of $X$; whence, they lie in $N_{\text {Aut } X}(G)$. Therefore,

$$
N_{\text {Aut } X}(G)=N_{\mathrm{GL}\left(V^{*}\right)}(G) \cap \operatorname{Tran}_{\mathrm{GL}\left(V^{*}\right)}(X, X) .
$$

From closedness of $G$ in $\operatorname{GL}\left(V^{*}\right)$ and that of $X$ in $V^{*}$ we deduce, respectively, that $N_{\mathrm{GL}\left(V^{*}\right)}(G)$ and $\operatorname{Tran}_{\mathrm{GL}\left(V^{*}\right)}(X, X)$ are closed in $\mathrm{GL}\left(V^{*}\right)$ (see [Bo91, Prop. 1.7]). This and (40) imply that $N_{\text {Aut } X}(G)$ is closed in $\operatorname{GL}\left(V^{*}\right)$. Hence $N_{\text {Aut } X}(G)$ is an algebraic subgroup of Aut $X$.

Theorem 5. Let $X$ be an irreducible affine variety and let $G$ be a reductive algebraic subgroup in Aut $X$ such that

$$
k[X]^{G}=k .
$$

In either of the following cases $N_{\mathrm{Aut} X}(G)$ is the algebraic subgroup of Aut $X$ :

(i) $G$ has a fixed point in $X$.

(ii) $G^{0}$ is semisimple.

Proof. Take $f \in k[X]$. We shall prove that in each of cases (i) and (ii) the $k$-linear span of the orbit $N_{\text {Aut } X}(G) \cdot f$ is finite dimensional. The claim of the theorem will then follow from Lemma 12.

Let $\mathcal{M}(G)$ be the set of isomorphism classes of algebraic simple $G$-modules. If $L$ is an algebraic $G$-module, denote by $L_{\mu}$ its isotypic component of type $\mu \in \mathcal{M}(G)$. 
Since $G$ is reductive, we have (see $[\mathrm{PoVi94}, 3.13]$ )

$$
k[X]=\bigoplus_{\mu \in \mathcal{M}(G)} k[X]_{\mu}
$$

The group $N_{\text {Aut } X}(G)$ permutes the isotypic components of the $G$-module $k[X]$.

Since $k[X]_{\mu}$ is a finitely generated $k[X]^{G}$-module (see [PoVi94, Thm. 3.24]), (41) implies that

$$
\operatorname{dim}_{k} k[X]_{\mu}<\infty \quad \text { for every } \mu .
$$

In view of $(42)$, there are elements $\mu_{1}, \ldots, \mu_{s} \in \mathcal{M}(G)$ such that

$$
k[X]_{\mu_{i}} \neq 0 \text { for all } i \text {, and } f \in k[X]_{\mu_{1}} \oplus \cdots \oplus k[X]_{\mu_{s}} .
$$

(i) Assume that in $X$ there is a $G$-fixed point $a$. Since closed orbits are separated by $G$-invariant regular functions (see [PoVi94, Thm.4.7]), it follows from (41) that there are no other $G$-fixed points in $X$. Hence $a$ is fixed by $N_{\text {Aut } X}(G)$ as well. Therefore, the ideal

$$
\mathfrak{m}_{a}:=\{f \in k[X] \mid f(a)=0\}
$$

is $N_{\text {Aut } X}(G)$-invariant. Hence every member of the decreasing filtration

$$
\mathfrak{m}_{a} \supset \cdots \supset \mathfrak{m}_{a}^{d} \supset \mathfrak{m}_{a}^{d+1} \supset \cdots .
$$

is $N_{\text {Aut } X}(G)$-invariant. This filtration has the property (see [AtMa69, Cor. 10.18]) that

$$
\bigcap_{d} \mathfrak{m}_{a}^{d}=0
$$

In view of (45), we have a decreasing system of nested linear subspaces $\left\{k[X]_{\mu} \cap \mathfrak{m}_{a}^{d} \mid d=1,2, \ldots\right\}$. Since they are finite dimensional (see (43)), there is $d_{\mu}$ such that $k[X]_{\mu} \cap \mathfrak{m}_{a}^{d}=k[X]_{\mu} \cap \mathfrak{m}_{a}^{d+1}$ for every $d \geqslant d_{\mu}$. From (46) it then follows that, in fact,

$$
k[X]_{\mu} \cap \mathfrak{m}_{a}^{d}=0 \text { for every } d \geqslant d_{\mu} .
$$

Let $l \in \mathbf{Z}, l \geqslant \max \left\{d_{\mu_{1}}, \ldots, d_{\mu_{s}}\right\}$. From (47) it follows that

$$
k[X]_{\mu_{i}} \cap \mathfrak{m}_{a}^{l}=0 \text { for every } i=1, \ldots, s .
$$

Since the natural projection $\pi: k[X] \rightarrow k[X] / \mathfrak{m}_{a}^{l}$ is an epimorphism of $G$-modules, we have $\pi\left(k[X]_{\mu}\right)=\left(k[X] / \mathfrak{m}_{a}^{l}\right)_{\mu}$ for every $\mu \in \mathcal{M}(G)$. Whereas

$$
\operatorname{dim}_{k} k[X] / \mathfrak{m}_{a}^{l}<\infty,
$$

(see [AtMa69, Prop. 11.4]), this implies finiteness of the set of $\mu \in \mathcal{M}(G)$ such that

$$
k[X]_{\mu} \neq 0 \quad k[X]_{\mu} \cap \mathfrak{m}_{a}^{l}=0 .
$$

Let $\left\{\lambda_{1}, \ldots, \lambda_{t}\right\}$ be this set. Since (49) holds for $\mu=\mu_{1}, \ldots, \mu_{s}$ (see (44), $(48))$, we may assume that

$$
\lambda_{i}=\mu_{i} \quad i=1, \ldots, s .
$$

Whereas the group $N_{\text {Aut } X}(G)$ permutes the isotypic components of the $G$ module $k[X]$ and sends $\mathfrak{m}_{a}^{l}$ into itself, it permutes $k[X]_{\lambda_{1}}, \ldots, k[X]_{\lambda_{t}}$. Hence 
$k[X]_{\lambda_{1}} \oplus \cdots \oplus k[X]_{\lambda_{t}}$ is an $N_{\text {Aut }} \mathbf{A}^{n}(G)$-invariant subspace of $k[X]$. In views of (43), (44), and (50), it is finite dimensional and contains $f$. Hence the $k$-linear span of the orbit $N_{\text {Aut } X}(G) \cdot f$ is finite dimensional, as claimed.

(ii) Let $G^{0}$ be semisimple. From the Weyl formula for dimension of simple $G^{0}$-module and finiteness of the index $\left[G: G^{0}\right]$ it follows that, up to isomorphism, there are only finitely many algebraic simple $G$-modules whose dimension does not exceed any preassigned constant. This implies finiteness of the set of $\mu \in \mathcal{M}(G)$ such that

$$
k[X]_{\mu} \neq 0 \quad \operatorname{dim}_{k} k[X]_{\mu} \leqslant \max _{i} \operatorname{dim}_{k} k[X]_{\mu_{i}} .
$$

Let $\left\{\lambda_{1}, \ldots, \lambda_{t}\right\}$ be this set. We may assume that (50) holds. Since $N_{\text {Aut } X}(G)$, permuting isotypic components, preserves their dimensions, $k[X]_{\lambda_{1}} \oplus \cdots \oplus$ $k[X]_{\lambda_{t}}$ is invariant with respect to $N_{\text {Aut } X}(G)$. The proof can be now completed as in case (i).

Corollary 9. Let $X$ and $G$ be the same as in Theorem 5 and let $k=\mathbf{C}$. Assume that the variety $X$ is simply connected and smooth, and $\chi(X)=1$. Then $N_{\text {Aut } X}(G)$ is an algebraic subgroup of Aut $X$.

Proof. By corollary of theorem on étale slice (see [Lu73, III, Cor. 2] and [PoVi94, Thm.6.7]), condition (41) and smoothness of $X$ imply that $X$ is a homogeneous vector bundle over the unique closed $G$-orbit $\mathcal{O}$ in $X$. Hence $\mathcal{O}$ is simply connected and $\chi(X)=\chi(\mathcal{O})$. Being affine, $\mathcal{O}$ is isomorphic to $G / H$ for some reductive subgroup $H$ (see [PoVi94, Thm. 4.17]). The conditions that $G / H$ is simply connected and $\chi(G / H)=1$ imply that $G=$ $H$ (see $[\mathrm{KrPo} 85,5.1]$ ). Hence $\mathcal{O}$ is a fixed point. The claim now follows from Theorem 5(i).

Corollary 10. Let $G$ be an reductive algebraic subgroups of Aut $\mathbf{A}^{n}$ and $k\left[\mathbf{A}^{n}\right]^{G}=k$. Then $N_{\mathrm{Aut}} \mathbf{A}^{n}(G)$ is the algebraic subgroup of Aut $\mathbf{A}^{n}$.

Proof. In view of char $k=0$, by the Lefschetz principle [Ha77, 15.1] we may assume that $k=\mathbf{C}$. Since $\mathbf{A}^{n}$ is simply connected and smooth, and $\chi\left(\mathbf{A}^{n}\right)=1$, the claim follows from Corollary 9 .

Remark 5. The following example shows that condition (41) alone (for irreducible affine $X$ and reductive $G$ ) does not, in general, imply that $N_{\text {Aut } X}(G)$ is algebraic.

Example 1. Let $G$ be an algebraic torus of dimension $n \geqslant 2$. Take as $X$ the underlying variety of the algebraic group $G$. Its group of automorphisms Aut $\operatorname{gr}_{\mathrm{r}} G$ is embedded in Aut $X$ and is isomorphic to $\mathrm{GL}_{n}(\mathbf{Z})$. The action of $G$ on $X$ by left translations embeds $G$ in Aut $X$. These two subgroups generate Aut $X$, more precisely, Aut $X=$ Aut $_{\mathrm{gr}} G \ltimes G$. Therefore, $N_{\text {Aut } X}(G)=$ Aut $X$. Let $g \in$ Aut $_{\mathrm{gr}} G$ be an element of infinite order and let $f_{1}, \ldots, f_{n} \in$ $k[X]$ be a base of $\mathrm{X}(G)$. Then $g^{d} \cdot f_{i} \in \mathrm{X}(G)$ for every $d \in \mathbf{Z}$ and $i=1, \ldots, n$, and the set $C_{i}:=\left\{g^{d} \cdot f_{i} \mid i \in \mathbf{Z}\right\}$ is finite if and only if the stabilizer of $f_{i}$ with respect to the cyclic group generated by $g$ is nontrivial. Assume that all $C_{1}, \ldots, C_{n}$ are finite. Then there is $d \in \mathbf{Z}, d \neq 0$, such that $g^{d} \cdot f_{i}=f_{i}$ for every $i=1, \ldots, n$. Since $f_{1}, \ldots, f_{n}$ is a base in $\mathrm{X}(G)$, this means that the 
autormorphism $g^{d}$ is trivial contrary to the assumption that the order of $g$ is infinite and $d \neq 0$. Hence $C_{i}$ is infinite for some $i$. Since different characters are linear independent over $k$ (see [Bo91, Lemma 8.1]), this implies that the $k$-linear span of the set $C_{i}$ (and hence of the orbit $N_{\text {Aut } X}(G) \cdot f_{i}$ ) is infinite dimensional.

We shall now use the obtained information in the proof of algebraicity of the groups $N_{\text {Aut }} \mathbf{A}^{n}\left(D_{n}\left(l_{1}, \ldots, l_{n}\right)\right)$.

Theorem 6. $N_{\text {Aut }} \mathbf{A}^{n}\left(D_{n}\left(l_{1}, \ldots, l_{n}\right)\right)$ for every $l_{1}, \ldots, l_{n}$ is an algebraic subgroup of Aut $\mathbf{A}^{n}$. Moreover,

(i) $N_{\mathrm{Aut}} \mathbf{A}^{n}\left(D_{n}\right)=N_{\mathrm{GL}_{n}}\left(D_{n}\right)$.

(ii) If $\left(l_{1}, \ldots, l_{n}\right) \neq(0, \ldots, 0)$, then

$$
N_{\mathrm{Aut}} \mathbf{A}^{n}\left(D_{n}\left(l_{1}, \ldots, l_{n}\right)\right) \subseteq N_{\mathrm{GL}_{n}}\left(D_{n}\right)
$$

in either of the following cases:

(a) all the numbers $l_{1}, \ldots, l_{n}$ are nonzero and have the same sign;

(b) none of the numbers $l_{1}, \ldots, l_{n}$ is equal to \pm 1 ;

(c) the numbers $l_{1}, \ldots, l_{n}$ contain 0 and \pm 1 , at least two of them are nonzero, and all nonzero of them have the same sign.

(iii) If $l_{i}=1$ and $l_{j}=0$ for $j \neq i$, then $N_{\text {Aut }} \mathbf{A}^{n}\left(D_{n}\left(l_{1}, \ldots, l_{n}\right)\right)$ is isomorphic to $N_{\mathrm{GL}_{n-1}}\left(D_{n-1}\right) \times \operatorname{Aff}_{1}$ and consists of all $\left(g_{1}, \ldots, g_{n}\right) \in \operatorname{Aff}_{n}$ of the form

$$
g_{j}= \begin{cases}t_{j} x_{\sigma(j)} & j \neq i \\ t_{j} x_{j}+s & j=i,\end{cases}
$$

where $t_{1}, \ldots, t_{n}, s \in k$ and $\sigma$ is a permutation of the set $\{1, \ldots, i-1, i+$ $1, \ldots, n\}$.

Proof. If $G$ is a subgroup of Aut $\mathbf{A}^{n}$ and $g \in N_{\text {Aut }} \mathbf{A}^{n}(G), a \in \mathbf{A}^{n}$, then

$$
\begin{aligned}
g(G \cdot a)= & G \cdot g(a), \quad g G_{a} g^{-1}=G_{g(a)}, \quad g(\overline{G \cdot a})=\overline{g(G \cdot a)}, \quad \text { and } \\
& \text { if } G \text { is algebraic, } \quad \operatorname{dim} G \cdot a=\operatorname{dim} g(G \cdot a) .
\end{aligned}
$$

Let $G=D_{n}$. Lemma $5(\mathrm{i})$ implies that $\bigcup_{i=1}^{n} H_{i}$ is the set of points whose $G$-stabilizer has positive dimension. By (53), it is $g$-invariant. Since the restriction of $g$ to the variety $\bigcup_{i=1}^{n} H_{i}$ is its automorphism, $g$ permutes its irreducible components $H_{1}, \ldots, H_{n}$, i.e., there is a permutation $\sigma \in S_{n}$ such that

$$
g\left(H_{i}\right)=H_{\sigma(i)} \quad \text { for every } i=1, \ldots, n .
$$

Since the ideal in $k\left[\mathbf{A}^{n}\right]$ determined by $H_{i}$ is generated by $x_{i}$, this shows that the polynomial $g^{*}\left(x_{i}\right)$ divides $x_{\sigma(i)}$, hence $g^{*}\left(x_{i}\right)=t_{i} x_{\sigma(i)}$ for some nonzero $t_{i} \in k$. Therefore, $g \in N_{\mathrm{GL}_{n}}\left(D_{n}\right)$ (see (3), (8)). This proves (i).

Further, let $G=D_{n}\left(l_{1}, \ldots, l_{n}\right),\left(l_{1}, \ldots, l_{n}\right) \neq(0, \ldots, 0)$.

Assume that condition (a) holds. Then (53) and Corollary of Lemma 7 imply that $g$ permutes the orbits $\mathcal{O}_{1}, \ldots \mathcal{O}_{n}$, i.e., there exists a permutation $\sigma \in S_{n}$ such that $g\left(\mathcal{O}_{i}\right)=\mathcal{O}_{\sigma(i)}$ for every $i$. In view of Lemma 6 and (53), this implies that $g$ has property (54), and as is shown above, the latter implies that $g \in N_{\mathrm{GL}_{n}}\left(D_{n}\right)$. Thus, (51) is proved in the case when condition (a) holds. 
Assume that condition (b) holds. Then $g$-invariance of $\bigcup_{i=1}^{n} H_{i}$ follows from (53) and Lemma 8. Now the same argument as for $G=D_{n}$ completes the proof of (51) in the case when condition (b) holds.

Assume that condition (c) holds. Proving (51), we may replace $G$ by the group conjugate to $G$ by means of an appropriate element of $N_{\mathrm{GL}_{n}}\left(D_{n}\right)$ and assume that (35) holds. The set $\left\{a \in \mathbf{A}^{n} \mid \operatorname{dim} G_{a}>0\right\}$ is closed (see [PoVi94, 1.4]). By virtue of (53), it is $g$-invariant, and Lemmas 5 and 10(ii) imply that its $(n-1)$-dimensional irreducible components are $H_{q+1}, \ldots$ $\ldots, H_{n}$. Hence $g$ permutes $H_{q+1}, \ldots, H_{n}$. Further, it follows from Lemmas 5 and 10 (ii) that $\mathcal{O}_{p+1}, \ldots, \mathcal{O}_{q}$ are all the $G$-orbits $\mathcal{O}$ in $\mathbf{A}^{n}$ such that $G_{a}$ is finite and nontrivial for $a \in \mathcal{O}$. From (53) it then follows that $g$ permutes the orbits $\mathcal{O}_{p+1}, \ldots, \mathcal{O}_{q}$ and, therefore, permutes their closures $H_{p+1}, \ldots, H_{q}$. Finally, in view of Lemmas 5 and 10(ii), all the $G$-orbits $\mathcal{O}$ in $\mathbf{A}^{n}$ such that $G_{a}$ is trivial for $a \in \mathcal{O}$ are exhausted by the orbits $\mathcal{O}_{1}, \ldots, \mathcal{O}_{p}$ and $G \cdot a$, where $a \notin \bigcup_{i=1}^{n} H_{i}$. Since $G$ is reductive, every $G$-orbit in $\mathbf{A}^{n}$ contains in its closure a unique closed $G$-orbit (see [PoVi94, Cor.p. 189]). By Lemma 6 , for each of the orbits $\mathcal{O}_{1}, \ldots, \mathcal{O}_{p}$ this closed orbit is the fixed point $(0, \ldots, 0)$. On the other hand, by Lemma 10(i), the closed orbit lying in $\overline{G \cdot a}$ for $a \notin \bigcup_{i=1}^{n} H_{i}$ has dimension $q-1 \geqslant 1$ and, therefore, is not the fixed point. Hence $g$ permutes $\mathcal{O}_{1}, \ldots, \mathcal{O}_{p}$, and therefore, permutes their closures $H_{1}, \ldots, H_{p}$. Thereby, it is proved that (54) holds for a certain permutation $\sigma \in S_{n}$. As above, this allows to conclude that (51) holds in case (c). Thus, (ii) is proved.

Assume now that $l_{i}=1$ and $l_{j}=0$ for $j \neq i$. From (53) and Lemma 11(i) it follows that the closed set $\bigcup_{j \neq i} H_{j}$ is invariant with respect to $g$. Hence $g$ permutes its irreducible components $H_{1}, \ldots, H_{i-1}, H_{i+1}, \ldots, H_{n}$. As above, from here we conclude that for $j \neq i$ the equality $g_{j}=t_{j} x_{\sigma(j)}$ holds for some $t_{j} \in k$ and some permutation $\sigma$ of the set $\{1, \ldots, i-1, i+1, \ldots, n\}$. Further, (53) and Lemma 11 imply that $g\left(\mathcal{O}_{i}\right)$ is an orbit open in a certain hyperplane $H(c)$ (see the notation in Lemma 11). Since $\overline{\mathcal{O}_{i}}=H_{i}$, and $x_{i}$ and $x_{i}+c$ are, respectively, the generators of the ideals of hyperplanes $H_{i}$ and $H(c)$, we conclude that $g_{i}=g^{*}\left(x_{i}\right)$ differs from $x_{i}+c$ only by a nonzero constant factor: $g_{i}=t_{i} x_{i}+s$ for some $t_{i}, s \in k, t_{i} \neq 0$. Thus, $g$ is of the form (52). Conversely, clearly, every element $g \in$ Aut $\mathbf{A}^{n}$ of the form (52) normalizes $G$. This proves (iii).

Finally, let us prove the first claim of this theorem. According to (i), (ii), and (iii), the group $N_{\text {Aut }} \mathbf{A}^{n}\left(D_{n}\left(l_{1}, \ldots, l_{n}\right)\right)$ is algebraic if either $\left(l_{1}, \ldots, l_{n}\right)=$ $(0, \ldots, 0)$, or $\left(l_{1}, \ldots, l_{n}\right) \neq(0, \ldots, 0)$ and any of conditions $(\mathrm{a}),(\mathrm{b}),(\mathrm{c})$ of statement (ii) or condition of statement (iii) hold. The only case not covered by these conditions is that when among $l_{1}, \ldots, l_{n}$ there are numbers with different signs. However, it follows from Lemma 9 that in this case there are no nonconstant $D_{n}\left(l_{1}, \ldots, l_{n}\right)$-invariant regular functions on $\mathbf{A}^{n}$. But then by Corollary 10 of Theorem 5 we conclude that $N_{\text {Aut }} \mathbf{A}^{n}\left(D_{n}\left(l_{1}, \ldots, l_{n}\right)\right)$ is algebraic. 


\section{Fusion theorems for tori in Aut $\mathbf{A}^{n}$ And Aut ${ }^{*} \mathbf{A}^{n}$}

Fusion theorems describe subgroups that control fusion of subsets under conjugation. Namely, let $G$ be an (abstract) groups and let $H$ be its subgroup. We say that $N_{G}(H)$ controls fusion of subsets in $H$ under conjugation by the elements of $G$, if the following property holds:

for any subset $S \subseteq H$ and an element $g \in G$ such that $g S g^{-1} \subseteq H$, there is an element $w \in N_{G}(H)$, such that $g s g^{-1}=w s w^{-1}$ for every element $s \in S$.

If property $(\mathrm{F})$ holds for a pair $(G, H)$, then we say that for $H$ in $G$ fusion theorem holds. Notice that the action of $N_{G}(H)$ on $H$ by conjugation boils down to the action of the "Weyl group" $N_{G}(H) / Z_{G}(H)$.

Examples 7. Fusion theorem for $H$ in $G$ holds in the following cases:

1. $G$ is a finite group and $H$ is its Abelian Sylow $p$-subgroup. It is the classical Burnside's result.

2. $G$ is an affine algebraic group and $H$ is its maximal torus. It is the classical result of the theory of algebraic groups, see, e.g., [Se00, 1.1.1].

3. $(G, H)=\left(\mathrm{Cr}_{n}, D_{n}\right)$. It is Serre's result [Se10, Thm. 1.1]. Since every $n$-dimensional torus in $\mathrm{Cr}_{n}$ is maximal and conjugate to $D_{n}$ (see Theorem 2(i),(ii)), here one can replace $D_{n}$ by any $n$-dimensional torus. Recall that for $n \geqslant 5$ in $\mathrm{Cr}_{n}$ there exist $(n-3)$-dimensional maximal tori (see Corollary 7 (iii)).

Question 8. Does fusion theorem hold if $D_{n}$ is replaced by such a torus?

We shall now prove that fusion theorem holds for $n$-dimensional tori in Aut $\mathbf{A}^{n}$ and $(n-1)$-dimensional tori in $\mathrm{Aut}^{*} \mathbf{A}^{n}$.

Lemma 13. For every element $g=\left(g_{1}, \ldots, g_{n}\right) \in$ Aut $\mathbf{A}^{n}$ there is an element $g^{\prime} \in \mathrm{SL}_{n}$ such that if $s, g s g^{-1} \in \mathrm{GL}_{n}$, then

$$
g s g^{-1}=g^{\prime} s g^{\prime-1} \text {. }
$$

Proof. Let $g_{i}=g_{i}^{(0)}+g_{i}^{(1)}+\ldots$, where $g_{i}^{(s)}$ is a form of degree $s$ in $x_{1}, \ldots, x_{n}$. Since $\operatorname{Jac}(g) \in k$, we have $\operatorname{Jac}(g)=\operatorname{det}\left(\partial g_{i} /\left.\partial x_{j}\right|_{x_{1}=\ldots=x_{n}=0}\right)$. But the righthand side of this equality is equal to $\operatorname{det}\left(\partial g_{i}^{(1)} / \partial x_{j}\right)$. Hence

$$
g^{(1)}:=\left(g_{1}^{(1)}, \ldots, g_{n}^{(1)}\right) \in \mathrm{GL}_{n} .
$$

The automorphism $g^{(1)}$ is the differential of the automorphism $g$ at the point $(0, \ldots, 0)$. From $(56)$ it follows that

$$
\begin{aligned}
& \text { (i) } g=g^{(1)} \text { if } g \in \mathrm{GL}_{n} \text {; } \\
& \text { (ii) }(g a)^{(1)}=g^{(1)} a \text { and }(a g)^{(1)}=a g^{(1)} \text { if } a \in \mathrm{GL}_{n} \text {. }
\end{aligned}
$$

Now let $s$ and $t:=g s g^{-1}$ be the elements of $\mathrm{GL}_{n}$. In view of (56), we have $g^{(1)} \in \mathrm{GL}_{n}$, and $g s=t g$ and (57) imply that $g^{(1)} s=t g^{(1)}$. Therefore, the product of $g^{(1)}$ by a constant $\alpha \in k$ such that $\alpha^{n} \operatorname{det} g^{(1)}=1$ can be taken as $g^{\prime}$.

Theorem 9. The following pairs $(G, H)$ have property $(\mathrm{F})$ : 
(i) (Fusion theorem for $n$-dimensional tori in Aut $\mathbf{A}^{n}$ )

$$
(G, H)=\left(\text { Aut } \mathbf{A}^{n} \text {, an n-dimensional torus }\right) .
$$

(ii) (Fusion theorem for $(n-1)$-dimensional tori in Aut $^{*} \mathbf{A}^{n}$ )

$$
(G, H)=\left(\text { Aut }^{*} \mathbf{A}^{n} \text {, an }(n-1) \text {-dimensional torus }\right) .
$$

Proof. (i) Let $G=$ Aut $\mathbf{A}^{n}$, let $H$ be a torus, and $\operatorname{dim} H=n$. Then, by Theorem 3 we may assume that $H$ is a maximal torus in $\mathrm{GL}_{n}$. Maintain the notation of the formulation of property $(\mathrm{F})$. By Lemma 13 there exists an element $g^{\prime} \in \mathrm{GL}_{n}$ such that for every element $s \in S$ equality (55) holds. Therefore, $g^{\prime} S g^{\prime-1}=g S g^{-1} \subseteq H$. Since $g^{\prime} \in \mathrm{GL}_{n}$, this shows that

$$
H^{\prime}:=g^{\prime-1} H g^{\prime}
$$

is another maximal torus in $\mathrm{GL}_{n}$ containing $S$. The tori $H$ and $H^{\prime}$ lie in the (closed [Bo91, I.1.7]) subgroup $Z_{\mathrm{GL}_{n}}(S)$ of the group $\mathrm{GL}_{n}$ and therefore, are maximal tori of $Z_{\mathrm{GL}_{n}}(S)$. In view of conjugacy of maximal tori in any affine algebraic group [Bo91, IV.11.3], there is an element

$$
z \in Z_{\mathrm{GL}_{n}}(S) \text {, }
$$

such that

$$
H^{\prime}=z H z^{-1} .
$$

From (58) and (60) it follows that $w:=g^{\prime} z \in N_{\mathrm{GL}_{n}}(H)$, and from (59) and (55) that $g s g^{-1}=w s w^{-1}$ for every $s \in S$. This proves (i).

(ii) The same argument applies in the case where $G=$ Aut $^{*} \mathbf{A}^{n}, H$ is a torus, and $\operatorname{dim} H=n-1$ : one only has to replace $\mathrm{GL}_{n}$ by $\mathrm{SL}_{n}$, the reference to Theorem 3 by the reference to Theorem 4, and notice that by Lemma 13 the element $g^{\prime}$ may be taken from $\mathrm{SL}_{n}$.

\section{Applications: The Classifichtions of Classes of COnjugate SUBGROUPS}

In this section, using the obtained results, we derive the classifications specified in the Introduction and, in particular, prove the generalizations to disconnected groups of the Białynicki-Birula's theorems on linearization of actions on $\mathbf{A}^{n}$ of tori of dimension $\geqslant n-1$.

Theorem 10. Let $G$ be an algebraic subgroups of Aut $\mathbf{A}^{n}$ such that $G^{0}$ is a torus.

(i) If $\operatorname{dim} G=n$ or $n-1$, then there is an element $g \in$ Aut $\mathbf{A}^{n}$ such that $g G g^{-1} \subset \mathrm{GL}_{n}$ and $g G^{0} g^{-1} \subset D_{n}$.

(ii) If $G \subset$ Aut* $^{*} \mathbf{A}^{n}$ and $\operatorname{dim} G=n-1$, then there is an element $g \in$ Aut* ${ }^{n}$ such that $g G g^{-1} \subset \mathrm{SL}_{n}$ and $g G^{0} g^{-1}=D_{n}^{*}($ see $(9))$.

Proof. (i) By virtue of conjugacy of maximal tori in $\mathrm{GL}_{n}$, it suffices to prove the existence of $g \in$ Aut $\mathbf{A}^{n}$ such that $g G g^{-1} \subset \mathrm{GL}_{n}$.

The group $G$ is reductive. Therefore, by Corollary of Theorem on étale slice [Lu73, Cor. 2, p. 98] the claim holds if $k\left[\mathbf{A}^{n}\right]^{G}=k$ (here only reductivity of $G$ is used, not the stronger condition that $G^{0}$ is a torus). Therefore, in 
what follows we may assume that $k\left[\mathbf{A}^{n}\right]^{G} \neq k$. The latter is equivalent to the condition

$$
k\left[\mathbf{A}^{n}\right]^{G^{0}} \neq k .
$$

In view of Theorem 3 and equality (13), replacing $G$ by a conjugate group, we may assume that

$$
G^{0}=D_{n}\left(l_{1}, \ldots, l_{n}\right) \subset \mathrm{GL}_{n} .
$$

The claim on the existence of $g$ will be proved if we show that (62) and (61) imply the inclusion

$$
G \subset \mathrm{GL}_{n} .
$$

Let $F$ be a finite subgroup of $G$ that intersects every connected component of this group - such a subgroup exists, see [BoSe64, Lemme 5.11]. Then

$$
G=F G^{0} .
$$

Since $G^{0}$ is a normal subgroup of $G$, we have $F \subset N_{\text {Aut }} \mathbf{A}^{n}\left(D_{n}\left(l_{1}, \ldots, l_{n}\right)\right)$. In view of Theorem 6 this shows that

$$
F \subset N_{\mathrm{GL}_{n}}\left(D_{n}\right) \subset \mathrm{GL}_{n}
$$

if either $\left(l_{1}, \ldots, l_{n}\right)=(0, \ldots, 0)$, or $\left(l_{1}, \ldots, l_{n}\right) \neq(0, \ldots, 0)$ and any of conditions (a), (b), (c) of statement (ii) of Theorem 6 holds. By virtue of (62) and (64), this proves (63) for the specified $\left(l_{1}, \ldots, l_{n}\right)$ 's. From Lemma 9 and (61) it now follows that it remains to consider the last possibility for $\left(l_{1}, \ldots, l_{n}\right)$, namely, that where $l_{i}=1$ and $l_{j}=0$ if $j \neq i$ for certain $i$.

Turning to its consideration, take some element $g \in F$. Then, by Theorem 6(iii), equalities (52) hold. Since $F$ is finite, the order of $g$ is finite. This implies that in (52) we have $s=0$. This and (8) mean that in the case under consideration (65) holds as well, and therefore, (63), too. This proves (i).

(ii) The proof is the same as that of (i) if Aut $\mathbf{A}^{n}$ is replaced by $\mathrm{Aut}^{*} \mathbf{A}^{n}$, $\mathrm{GL}_{n}$ by $\mathrm{SL}_{n}=\mathrm{GL}_{n} \bigcap \mathrm{Aut}^{*} \mathbf{A}^{n}, D_{n}\left(l_{1}, \ldots, l_{n}\right)$ by $D_{n}^{*}$, the reference to Theorem 3 by the reference to Theorem 4 , and it is taken into account that $D_{n}^{*}$ is the maximal torus of $\mathrm{SL}_{n}$.

Theorem 11 (Classification of $n$-dimensional diagonalizable subgroups of Aut $\mathbf{A}^{n}$ up to conjugacy in Aut $\mathbf{A}^{n}$ ). Up to conjugacy in Aut $\mathbf{A}^{n}$, the torus $D_{n}$ is the unique $n$-dimensional diagonalizable subgroup of Aut $\mathbf{A}^{n}$.

Proof. By virtue of Theorem 10 this follows from the fact that in $\mathrm{GL}_{n}$ every diagonalizable subgroup is conjugate to a subgroup of the torus $D_{n}$ (see [Bo91, p. 112, Prop.(d)]).

Theorem 12 (Classification of $(n-1)$-dimensional diagonalizable subgroups of Aut* $\mathbf{A}^{n}$ up to conjugacy in $\left.\mathrm{Aut}^{*} \mathbf{A}^{n}\right)$. Up to conjugacy in $\mathrm{Aut}^{*} \mathbf{A}^{n}$, the torus $D_{n}^{*}$ is the unique $(n-1)$-dimensional diagonalizable subgroup of Aut* $^{*} \mathbf{A}^{n}$.

Proof. By virtue of Theorem 10, this follows form the fact that in $\mathrm{SL}_{n}$ every diagonalizable subgroup is conjugate to a subgroup of the torus $D_{n}^{*}$ (this easily follows from [Bo91, p. 112, Prop.(d)]). 
Theorem 13 (Classification up to conjugacy in Aut $\mathbf{A}^{n}$ of maximal $n$-dimensional algebraic subgroups $G$ of Aut $\mathbf{A}^{n}$ such that $G^{0}$ is a torus). Up to conjugacy in Aut $\mathbf{A}^{n}$, the group $N_{\mathrm{GL}_{n}}\left(D_{n}\right)$ (see (8)) is the unique maximal algebraic subgroup of Aut $\mathbf{A}^{n}$ whose connected component of identity is an $n$-dimensional torus.

Proof. By virtue of Theorem 10, this follows from the fact that $D_{n}=$ $N_{\mathrm{GL}_{n}}\left(D_{n}\right)^{0}$.

Theorem 14 (Classification up to conjugacy in Aut* $\mathbf{A}^{n}$ of maximal $(n-1)$ dimensional algebraic subgroups $G$ in Aut* $\mathbf{A}^{n}$ such that $G^{0}$ is a torus). $U p$ to conjugacy in Aut* $^{*} \mathbf{A}^{n}$,

$$
N_{\mathrm{SL}_{n}}\left(D_{n}^{*}\right)=N_{\mathrm{GL}_{n}}\left(D_{n}\right) \cap \mathrm{SL}_{n}
$$

is the unique maximal algebraic subgroup of $\mathrm{Aut}^{*} \mathbf{A}^{n}$ whose connected component of identity is an $(n-1)$-dimensional torus.

Proof. By virtue of Theorem 10, this follows from the fact that $D_{n}^{*}=$ $N_{\mathrm{SL}_{n}}\left(D_{n}^{*}\right)^{0}$.

Denote by $\mathcal{L}_{n}$ the additive monoid of all $\left(l_{1}, \ldots, l_{n}\right) \in \mathbf{Z}^{n}$ such that

(a) $l_{1} \leqslant \cdots \leqslant l_{n}$;

(b) $\left(l_{1}, \ldots, l_{n}\right) \leqslant\left(-l_{n}, \ldots,-l_{1}\right)$ with respect to the lexicographical order on $\mathbf{Z}^{n}$.

Theorem 15 (Classification of $(n-1)$-dimensional diagonalizable subgroups in Aut $\mathbf{A}^{n}$ up to conjugacy in Aut $\mathbf{A}^{n}$ ).

(i) Every (n-1)-dimensional diagonalizable subgroup of the group Aut $\mathbf{A}^{n}$ is conjugate in Aut $\mathbf{A}^{n}$ to a unique subgroup of the form

$$
D_{n}\left(l_{1}, \ldots, l_{n}\right), \quad\left(l_{1}, \ldots, l_{n}\right) \in \mathcal{L}_{n} \backslash\{(0, \ldots, 0)\} .
$$

(ii) Every subgroup (66) is an $(n-1)$-dimensional diagonalizable group.

Proof. Let $G$ be an $(n-1)$-dimensional diagonalizable subgroup of Aut $\mathbf{A}^{n}$. Taking into account Theorem 10(i), conjugacy in $\mathrm{GL}_{n}$ of every diagonalizable subgroup of this group to a subgroup of the torus $D_{n}$, and Corollary 2(ii), we conclude that $G$ is conjugate in Aut $\mathbf{A}^{n}$ to a certain subgroup $D_{n}\left(l_{1}, \ldots, l_{n}\right)$ with $\left(l_{1}, \ldots, l_{n}\right) \neq(0, \ldots, 0)$. It follows from the definition of $\mathcal{L}_{n}$ and Corollary 2(iv) that we may presume that $\left(l_{1}, \ldots, l_{n}\right) \in \mathcal{L}_{n}$. Assume that $G$ is also conjugate in Aut $\mathbf{A}^{n}$ to a subgroup $D_{n}\left(l_{1}^{\prime}, \ldots, l_{n}^{\prime}\right)$ with $\left(l_{1}^{\prime}, \ldots, l_{n}^{\prime}\right) \in \mathcal{L}_{n}$. Then $D_{n}\left(l_{1}, \ldots, l_{n}\right)$ and $D_{n}\left(l_{1}^{\prime}, \ldots, l_{n}^{\prime}\right)$ are conjugate in Aut $\mathbf{A}^{n}$, and therefore, Theorem 9(i) implies that they are conjugate in $N_{\mathrm{GL}_{n}}\left(D_{n}\right)$. In view of Corollary 2(iv) and Definition $\mathcal{L}_{n}$, this implies that $\left(l_{1}, \ldots, l_{n}\right)=\left(l_{1}^{\prime}, \ldots, l_{n}^{\prime}\right)$. This proves (i).

Statement (ii) follows from Corollary 2(ii).

From Theorems 6, 11, and 15 we deduce

Theorem 16. If $G$ is a diagonalizable subgroup of Aut $\mathbf{A}^{n}$ of dimension $\geqslant n-1$, then $N_{\mathrm{Aut} \mathbf{A}^{n}}(G)$ is an algebraic subgroup of Aut $\mathbf{A}^{n}$. 
Remark 6 . It is easy to see that $n-1$ in Theorem 16 cannot be replaced by a smaller integer.

Theorem 17 (Classification of $(n-1)$-dimensional diagonalizable subgroups of Aut $\mathbf{A}^{n}$ up to conjugacy in $\left.\mathrm{Cr}_{n}\right)$.

(i) Two diagonalizable $(n-1)$-dimensional subgroups of the group Aut $\mathbf{A}^{n}$ are conjugate in $\mathrm{Cr}_{n}$ if and only if they are isomorphic.

(ii) Every (n-1)-dimensional diagonalizable subgroup of the group Aut $\mathbf{A}^{n}$ is conjugate in $\mathrm{Cr}_{n}$ to a unique closed subgroup of $D_{n}$ of the form

$$
\operatorname{ker} \varepsilon_{n}^{d}, \quad d \in \mathbf{Z}
$$

Proof. This follows from Theorems 15 and 1 and Corollary 2(i).

For $n \leqslant 3$, Theorems 2(i), 11, and 15 yield the classification of all tori in Aut $\mathbf{A}^{n}$ up to conjugacy in Aut $\mathbf{A}^{n}$ except for one-dimensional tori in Aut $\mathbf{A}^{3}$. The classification of the latter is given below in Theorem 18 .

Theorem 18 (Classification of one-dimensional tori in Aut $\mathbf{A}^{3}$ up to conjugacy in Aut $\left.\mathbf{A}^{3}\right)$. Every one-dimensional torus in Aut $\mathbf{A}^{3}$ is conjugate in Aut $\mathbf{A}^{3}$ to a unique torus of the form $T\left(l_{1}, l_{2}, l_{3}\right)$, where $\left(l_{1}, l_{2}, l_{3}\right) \in \mathcal{L}_{3}$.

Proof. Let $G$ be a one-dimensional torus in Aut $\mathbf{A}^{3}$. By Theorem 3 it is conjugate in Aut $\mathbf{A}^{3}$ to some torus $T\left(l_{1}, l_{2}, l_{3}\right)$. In view of $(8)$ and the equality $T\left(l_{1}, l_{2}, l_{3}\right)=T\left(-l_{1},-l_{2},-l_{3}\right)$, we may presume that $\left(l_{1}, l_{2}, l_{3}\right) \in \mathcal{L}_{3}$. Assume that $G$ is conjugate in Aut $\mathbf{A}^{3}$ to another torus $T\left(l_{1}^{\prime}, l_{2}^{\prime}, l_{3}^{\prime}\right)$ with $\left(l_{1}^{\prime}, l_{2}^{\prime}, l_{3}^{\prime}\right) \in \mathcal{L}_{3}$. Then $T\left(l_{1}, l_{2}, l_{3}\right)$ and $T\left(l_{1}^{\prime}, l_{2}^{\prime}, l_{3}^{\prime}\right)$ are conjugate in Aut $\mathbf{A}^{3}$, and therefore, by Theorem $9(\mathrm{i})$, also in $N_{\mathrm{GL}_{3}}\left(D_{3}\right)$. From this, Corollary 2(iv), the definition of $\mathcal{L}_{n}$, and Lemma 4 , it is then not difficult to deduce that $\left(l_{1}, l_{2}, l_{3}\right)=\left(l_{1}^{\prime}, l_{2}^{\prime}, l_{n}^{\prime}\right)$.

\section{JoRdAN DECOMPOSITION IN $\mathrm{Cr}_{n}$. TORSION PRIMES FOR THE Cremona groups}

Although the Cremona groups are infinite dimensional (this has a precise meaning, see [Ra64]), the analogies between them and algebraic groups strike the eye: they have the Zariski topology, algebraic subgroups, tori, roots, the Weyl groups, ... In [Se10, 1.2] Serre writes about the analogy

"groupe de Cremona de rang $n \longleftrightarrow$ groupe semi-simple de rang $n$ ".

Below we briefly touch upon two topics demonstrating that these analogies extend further. The second of them is intimately related to tori in the Cremona groups.

\section{Jordan decomposition in $\mathbf{C r}_{\boldsymbol{n}}$.}

Let $X$ be an algebraic variety. "Algebraic families" endow Bir $X$ with the Zariski topology [Ra64], [Bl10, 2], [Se10, 1.6]: a subset of Bir $X$ is closed if and only if its inverse image for every algebraic family $S \rightarrow \operatorname{Bir} X$ is closed. For every algebraic subgroup $G$ in $\operatorname{Bir} X$ and its subset $Z$, the closures of $Z$ in this topology and in the Zariski topology of the group $G$ coincide. In particular, $G$ is closed in $\operatorname{Bir} X$. 
Let us call an element $g \in \operatorname{Bir} X$ algebraic if in $\mathrm{Cr}_{n}$ there is an algebraic subgroup $G$ containing $g$. This is equivalent to the property that the closure of the cyclic group generated by $g$ is an algebraic group. If $G$ is affine, then for $g$ the Jordan decomposition in $G$ is defined, see [Bo91, Chap. I, §4]:

$$
g=g_{s} g_{n}
$$

In fact, $g_{s}$ and $g_{n}$ depend only of $g$, not of the choice of $G$. Indeed, let $G^{\prime}$ be another affine algebraic subgroup of $\operatorname{Bir} X$ containing $g$, and let $g=g_{s}^{\prime} g_{n}^{\prime}$ be the Jordan decomposition in $G^{\prime}$. Since $G \cap G^{\prime}$ is a closed subgroup of $G$ and of $G^{\prime}$, there exists the Jordan decomposition $g=g_{s}^{\prime \prime} g_{n}^{\prime \prime}$ in $G \cap G^{\prime}$. Applying theorem on behaviour of the Jordan decompositions under homomorphisms (see [Bo91, Thm. 4.4(4)]) to the embeddings $G \hookleftarrow G \cap G^{\prime} \hookrightarrow G^{\prime}$, we obtain $g_{s}=g_{s}^{\prime \prime}, g_{n}=g_{n}^{\prime \prime}, g_{s}^{\prime}=g_{s}^{\prime \prime}, g_{n}^{\prime}=g_{n}^{\prime \prime}$. Hence, if we call (67) the Jordan decomposition in $\operatorname{Bir} X$, we get the well-defined notion.

According to [Ma63], every algebraic subgroup of $\mathrm{Cr}_{n}$ is affine. Therefore, in $\mathrm{Cr}_{n}$ every algebraic element admits the Jordan decomposition.

Jordan decompositions in algebraic groups have several known properties [St65], [Bo91], for instance:

(a) Every semisimple element of a connected group lies in its torus.

(b) The set of all unipotent elements is closed.

(c) The conjugacy class of every semisimple element of a connected reductive group is closed.

(d) The closure of the conjugacy class of every element $g$ of a connected reductive group contains $g_{s}$.

Recall that $\mathrm{Cr}_{n}$ is connected and, for $n=1,2$, simple [Bl10], and that Aut $\mathbf{A}^{n}$, Aut ${ }^{*} \mathbf{A}^{n}$ are connected and Aut $^{*} \mathbf{A}^{n}$ is simple [Sh66], [Sh82]. It is natural to ask:

Question 19. Are there analogues or modifications of the mentioned properties for the groups $\mathrm{Cr}_{n}$, Aut $\mathbf{A}^{n}$, and $\mathrm{Aut}^{*} \mathbf{A}^{n}$ ?

For instance, property (a) for $\mathrm{Cr}_{n}$ holds if $n=1$, but does not hold if $n>1$. Indeed, if $n>2$, then by [Po12, Thm. 4.3] in $\mathrm{Cr}_{n}$ there is a semisimple element of order two that is not contained in any connected algebraic subgroup of the group $\mathrm{Cr}_{n}$. If $n=2$, then by virtue of Theorem 2(ii) and Corollary 5, all the elements of order $d<\infty$ in $\mathrm{Cr}_{n}$ that can be included in tori constitute a single conjugacy class, while, for instance, for even $d$, the set of all elements of order $d$ is the union of infinitely many conjugacy classes [Bl07]. On the other hand, in the groups Aut $\mathbf{A}^{2}$ and $A u t^{*} \mathbf{A}^{2}$ property (a) holds, because action of every finite group on $\mathbf{A}^{2}$ is linearizable [ $\left.\operatorname{Ig} 77\right]$.

\section{Torsion primes for the Cremona groups.}

Let $G$ be a connected reductive algebraic group and let $p$ be a prime integer. Recall (see $[\mathrm{Se} 00,1.3]$ and references therein) that $p$ is called torsion prime for the group $G$ if in $G$ there is a finite Abelian $p$-subgroup not contained in any torus of the group $G$. The set Tors $(G)$ of all torsion primes for the group $G$ admits various interpretations. For instance, $p \in \operatorname{Tors}(G)$ 
for $k=\mathbf{C}$ if and only if $\bigoplus_{i} \mathrm{H}_{i}(G, \mathbf{Z})$ contains an element of order $p$ (this explains the name). Finding Tors $(G)$ is reduced to the case where $G$ is simply connected. For every simple $G$ the set $\operatorname{Tors}(G)$ is explicitly described.

The possibility to speak about tori in groups of the form $\operatorname{Bir} X$ where $X$ is an algebraic variety, makes it possible to replace $G$ in the above definition by Bir $X$ or its subgroup thereby obtaining the well-defined notion. In particular, we consider what is obtained when $G$ is replaced by $\mathrm{Cr}_{n}$, Aut $\mathbf{A}^{n}$, or Aut $^{*} \mathbf{A}^{n}$ as the definitions of torsion primes for these groups. Denote the sets of these primes respectively by $\operatorname{Tors}\left(\mathrm{Cr}_{n}\right)$, Tors $\left(\operatorname{Aut} \mathbf{A}^{n}\right)$, and $\operatorname{Tors}\left(\operatorname{Aut}{ }^{*} \mathbf{A}^{n}\right)$. It is natural to ask

Question 20. What are, explicitly, the sets $\operatorname{Tors}\left(\mathrm{Cr}_{n}\right)$, $\operatorname{Tors}\left(\operatorname{Aut} \mathbf{A}^{n}\right)$, and Tors $\left(\right.$ Aut $\left.^{*} \mathbf{A}^{n}\right)$ ?

(About Tors $\left(\mathrm{Cr}_{n}\right)$ this question has been formulated and discussed in talk [Po12].)

Since $\mathrm{Cr}_{1}=\mathrm{PGL}_{2}$, we have

$$
\operatorname{Tors}\left(\mathrm{Cr}_{1}\right)=\{2\} \text {. }
$$

According to [Bl07], for every $d=2,3$, and 5 , in $\mathrm{Cr}_{2}$ there are infinitely many conjugacy classes of elements of order $d$. On the other hand, as is explained at the end of section on Jordan decomposition, in $\mathrm{Cr}_{2}$ there is the unique conjugacy class of elements of order $d$ contained in tori. Hence 2,3 , and 5 are torsion primes for the group $\mathrm{Cr}_{2}$. Consider a prime integer $p>5$. According to [Fe04, Thm. E], every element $g \in \mathrm{Cr}_{2}$ of order $p$ lies in a subgroup isomorphic to Aut $\mathbf{P}^{2}=\mathrm{PGL}_{3}$. Hence $g$ lies in a torus. Finally, according to $\left[\mathrm{BlO6}_{2}\right.$, Thm. B, p. 146], in $\mathrm{Cr}_{2}$ there is a unique up to conjugacy noncyclic finite Abelian p-group (it is denoted by 0.mn), and this group is contained in a maximal torus of a subgroup isomorphic to $\left(\text { Aut }\left(\mathbf{P}^{1} \times \mathbf{P}^{1}\right)\right)^{0}=$ $\mathrm{PGL}_{2} \times \mathrm{PGL}_{2}$. Thus we conclude that the torsion primes for $\mathrm{Cr}_{2}$ are the same as that for the exceptional simple algebraic group $\mathrm{E}_{8}$ :

$$
\operatorname{Tors}\left(\mathrm{Cr}_{2}\right)=\{2,3,5\} \text {. }
$$

From Aut $\mathbf{A}^{1}=$ Aff $_{1}$ and [ $\left.\operatorname{Ig} 77\right]$ it follows that every finite subgroup of Aut $\mathbf{A}^{n}$ (respectively, Aut* $\mathbf{A}^{n}$ ) for $n \leqslant 2$ is contained in a subgroup isomorphic to $\mathrm{GL}_{n}$ (respectively, $\mathrm{SL}_{n}$ ). Therefore,

$$
\operatorname{Tors}\left(\operatorname{Aut} \mathbf{A}^{n}\right)=\operatorname{Tors}\left(\mathrm{Aut}^{*} \mathbf{A}^{n}\right)=\{\varnothing\} \text { for } n \leqslant 2 \text {. }
$$

For $n \geqslant 3$, there is no comprehensive information about the sets $\operatorname{Tors}\left(\mathrm{Cr}_{n}\right)$, Tors $\left(\right.$ Aut $\mathbf{A}^{n}$ ), and Tors $\left(\right.$ Aut $^{*} \mathbf{A}^{n}$ ). From [Po12, Thm.4.3], (68), and (69) it follows that

$$
\operatorname{Tors}\left(\mathrm{Cr}_{n}\right)=\{2, \ldots\} \text { for every } n \text {. }
$$

According to [Be07], in $\mathrm{Cr}_{2}$ there is a 3-elementary Abelian subgroups of rank 3 (i.e., isomorphic to $\left.\left(\boldsymbol{\mu}_{d}\right)^{3}\right)$. Since $\mathrm{Cr}_{1}$ contains a cyclic subgroup of order 3 , and the direct product of $\mathrm{Cr}_{2}$ and $n-2$ copies of $\mathrm{Cr}_{1}$ can be embedded in $\mathrm{Cr}_{n}$ for $n \geqslant 3$, this implies that in $\mathrm{Cr}_{n}$ there is a 3-elementary Abelian subgroup $G$ of rank $n+1$. But from Lemma 2(i) it follows that for every prime integer $p$, the rank of any elementary Abelian $p$-subgroup of an 
$r$-dimensional torus is at most $r$. From this and Theorem 2(i) it follows that $G$ is not contained in a torus of $\mathrm{Cr}_{n}$. In view of (69) and (70), this yields

$$
\operatorname{Tors}\left(\mathrm{Cr}_{n}\right)=\{2,3, \ldots\} \text { for any } n \geqslant 2 \text {. }
$$

Question 21. What is the minimal $n$ such that 7 lies in one of the sets $\operatorname{Tors}\left(\mathrm{Cr}_{n}\right)$, Tors $\left(\operatorname{Aut} \mathbf{A}^{n}\right)$, and Tors $\left(\mathrm{Aut}^{*} \mathbf{A}^{n}\right)$ ?

Question 22. Are these sets finite?

\section{REFERENCES}

[AtMa69] M. F. Atiyah, I. G. MacDonald, Introduction to Commutative Algebra, Addison-Wesley, Ma, 1969.

[Be07] A. BeAuville, p-elementary subgroups of the Cremona group, J. Algebra 314 (2007), no. 2, 553-564.

[B-B66] A. BiAŁYNiCKI-Birula, Remarks on the action of an algebraic torus on $k^{n}$, Bull. Acad. Polon. Sci. Sér. Sci. Math. Astronom. Phys. 14 (1966), 177-181.

[B-B67] A. BiAŁYNICKI-BiRula, Remarks on the actionof an algebraic torus on $k^{n}$, II, Bull. Acad. Polon. Sci. Sér. Sci. Math. Astronom. Phys. 15 (1967), 123125.

[Bl06 1 J. BlAnc, Conjugacy classes of affine automorphisms of $\mathbf{K}^{n}$ and linear automorphisms of $\mathbf{P}^{n}$ in the Cremona groups, Manuscripta Math. 119 (2006), no. 2, 225-241.

[Bl06 2$]$ J. Blanc, Finite abelian subgroups of the Cremona group of the plane, Thèse No. 3777, Université de Genève, Faculté des Sciences, 2006.

[B107] J. Blanc, The number of conjugacy classes of elements of the Cremona group of some given finite order, Bull. Soc. Math. France 135 (2007), no. 3, 419-434.

[Bl10] J. Blanc, Groupes de Cremona, connexité et simplicité, Ann. Sci. Éc. Norm. Supér. (4) 43 (2010), no. 2, 357-364.

[Bo91] A. Borel, Linear Algebraic Groups, 2nd ed., Springer-Verlag, New York, 1991.

[BoSe64] A. Borel, J.-P. SERRE, Théorèmes de finitude en cohomoplogie galoisienne, Comm. Math. Helv. 39 (1964), 111-164.

[De70] M. Demazure, Sous-groupes algébriques de rang maximum du groupe de Cremona, Ann. Sci. de l'E.N.S., 4e sér. 3 (1970), fasc. 4, 507-588.

[Fe04] T. DE FERnex, On planar Cremona maps of prime order, Nagoya Math. J. 174 (2004), 1-28.

[Ha77] J. HARRIs, Algebraic Geometry, Graduate Texts in Mathematics, Vol. 133, Springer, New Yorks, 1992.

[Ig77] T. IGARASHI, Finite subgroups of the automorphism group of the affine plane, M.A. thesis, Osaka University, 1977.

[KKM-LR97] S. Kaliman, M. Koras, L. Makar-Limanov, P. Russell, $\mathbb{C}^{*}$-actions on $\mathbb{C}^{3}$ are linearizable, Electron. Res. Announc. Amer. Math. Soc. 3 (1997), 63-71 (electronic).

[KrPo85] H. Kraft, V. L. Popov, Semisimple group actions on the three dimensional affine space are linear, Comment. Math. Helvetici 60 (1985), 466-479.

[La81] G. Laumon, Comparaison de caracteristiques d'Euler-Poincaré en cohomologie l-adique, C. R. Acad. Sci. Paris 292 (1981), 209-212.

[Li11] A. Liendo, Roots of the affine Cremona group, Transform. Groups 16 (2011), no. 4, 1137-1142.

[Lu73] D. LunA, Slices étales, Bull. Soc. Math. France 33 (1973), 81-105.

[MaMi64] M. Marcus, H. Minc, A Survey of Matrix Theory and Matrix Inequalities, Allyn and Bacon, Boston, 1964. 
[Ma63] H. Matsumura, On algebraic groups of birational transformations, Atti Accad. Naz. Lincei Rend. Cl. Sci. Fis. Mat. Natur. 8 (1963), 34, 151-155.

[Po70] V. L. Popov, Stability criteria for the action of a semisimple group on a factorial manifold, Math. USSR Izv. 4 (1970), 527-535.

[Po051] V. L. Popov, Problems for problem session, arXiv:0504517v1 25 Apr2005.

$\left[\mathrm{Po}_{2}\right] \quad$ V. L. Popov, Roots of the affine Cremona group, in: Affine Algebraic Geometry, Seville, Spain, June 18-21, 2003, Contemporary Mathematics, Vol. 369, American Mathematical Society, Providence, RI, 2005, pp. 12-13.

[Po11] V. L. Popov, Some subgroups of the Cremona groups, to appear in: Proceedings of Conference on Affine Algebraic Geometry, Osaka, March 3-6, 2011, World Scientific, arXiv:1110. 2410v1 11 0ct2011.

[Po12] V. L. Popov, Tori in the Cremona groups, Talk delivered at the International workshop Essential Dimension and Cremona Groups, Chern Institute of Mathematics, Nankai University, Tianjin, China, June 11-15, 2012, http://www .nim.nankai.edu.cn/activites/conferences/hy20120611/index.htm.

[PoVi94] V. L. Popov, E. B. Vinberg, Invariant theory, in: Algebraic Geometry IV, Encyclopaedia of Mathematical Sciences, Vol. 55, Springer-Verlag, Berlin, 1994, pp. 123-284.

[Ra64] C. P. Ramanujam, A note on automorphism groups of algebraic varieties, Math. Ann. 156 (1964), 25-33.

[Ro56] M. Rosenlicht, Some basic theorems on algebraic groups, Amer. J. Math. 78 (1956), 401-443.

[Se55] J.-P. SERRE, Faisceaux algébriques cohérents, Ann. of Math. 61 (1955), $197-278$.

[Se00] J.-P. SERRE, Sous-groupes finis des groupes de Lie, in: Séminaire N. Bourbaki 1998/99, Exp.no. 864, Astérisque, Vol. 266, Société Mathématique de France, 2000, pp. 415-430.

[Se10] J.-P. SERRE, Le groupe de Cremona et ses sous-groupes finis, in: Séminaire N. Bourbaki 2008/09, Exp. no.1000, Astérisque, Vol.332, Société Mathématique de France, 2010, pp. 75-100.

[St65] R. SteInBerG, Regular elements of semisimple algebraic groups, Inst. Hautes Études Sci. Publ. Math. 25 (1965), 49-80.

[Sh66] I. R. Shafarevich, On some infinite-dimensional groups, Rend. Mat. e Appl. (5) 25 (1966), nos. 1-2, 208-212.

[Sh82] I. R. Shafarevich, On some infinite-dimensional groups. II, Mathematics of the USSR-Izvestiya 18 (1982), no. 1, 185-194.

[ShRe09] И. Р. ШАфАРЕВИЧ, А. О. РЕмИзОВ, Линейная алгебра и геометрия, Физматлит, M., 2009. [I. R. Shafarevich, A. O. Remizov, Linear Algebra and Geometry, Fizmatlit, Moscow, 2009 (in Russian).]

[Vi03] E. B. Vinberg, A Course in Algebra, Graduate Studies in Mathematics, Vol. 56, American Mathematical Society, Providence, RI, 2003.

Steklov Mathematical Institute, Russian Academy of Sciences, Gubkina 8, Moscow, 119991, Russia

E-mail address: popovvl@mi.ras.ru 